Beyond average: Using face regression to study social perception

Alex L. Jones

Swansea University

Author Note

Corresponding author:

Alex Jones,

Department of Psychology

Swansea University

Singleton Park

Swansea, SA2 8PP

Email: alexjonesphd@gmail.com

The authors declare that there are no potential conflicts of interest with respect to the research, authorship, and/or publication of this article. 
Understanding in the field of face perception is borne from advances in computer graphics techniques. Here, a new and fully data-driven algorithm is introduced for studying the social perception of the face, termed face regression. Given a set of photographs representing facial texture, coordinates delineating facial shape, and measured social traits, the algorithm learns relationships between each dimension of the faces (pixel values and coordinate points) and their associated social traits. Using the learned weights, the algorithm is capable of predicting faces of any score for any or all of the modelled traits, allowing for a fine-grained examination of facial features associated with social traits when compared to common facial averaging and transforming methods. In two applications, the algorithm addresses theoretical concerns with common methodologies in face perception, and demonstrates its utility through its ability to recreate face averages, and isolate the actual appearances associated with social traits. Perceptual validation experiments indicate participant reactions differ between composite images and those generated through face regression, giving novel insight to the function of social perception. A dedicated software package for utilising the algorithm is introduced, and future applications are discussed. 
The human face is one of the most important stimuli we encounter. From a single glance, we are able to infer a wealth of valuable social information, including an individuals' identity (Sheehan \& Nachman, 2014), sex (Farkas, 1987; Russell, 2009), age (George \& Hole, 1995), emotional state (Vuilleumier, Armony, Driver, \& Dolan, 2003), attractiveness (Rhodes, 2006), and health (Jones, 2018; Zebrowitz \& Rhodes, 2004). This information is signalled from the complex interplay between the structure and surface reflectance properties of faces (O'Toole, Price, Vetter, Bartlett, \& Blanz, 1999).

The vast literature on face perception has largely been made possible through the advance of computer graphics techniques and application of advanced statistical approaches. For example, the process of facial averaging, which has seen extensive application both within and beyond psychology, has improved dramatically from its first introduction. Initially, face images were aligned by pupil and mouth-centre locations before a simple average of greyscale pixel intensities were computed (Langlois \& Roggman, 1990). Modern approaches utilise algorithms to divide face images into subsets of Delaunay triangles, that are individually transformed to a new shape and texture using affine warping and pixel interpolation (Tiddeman, Burt, \& Perrett, 2001). Using these algorithms, faces can be warped to the average landmark configuration of a set of faces, before mathematically averaging pixel intensities, yielding well-defined and photorealistic composite images. This process can also be easily extended to create image warps of facial shape, skin texture, or colour. Given a pair of facial appearances, or 'anchors', and their associated landmark configurations, a third face can be transformed along the difference between the initial pair by a weighting factor, creating a series of 'steps' or a simple blend between the two. These applications have seen widespread use in psychological research, investigating 
attractiveness preferences between female and male average face shapes (Perrett et al., 1998), the role of cosmetics and skin colour on social judgments (Jones, Kramer, \& Ward, 2014; Stephen, Coetzee, \& Perrett, 2011), and as a method to test discrimination thresholds of properties that differ between the anchored faces when presented as warped gradations (Davies-Thompson et al., 2017), to illustrate but a few examples.

The above two approaches - facial averaging and transforming - are easily employed by researchers despite their relative computational complexity, thanks to dedicated programs such as Psychomorph (Burt \& Perrett, 1995; Tiddeman et al., 2001) and InterFace (Kramer, Jenkins, \& Burton, 2017). This availability has led to a wealth of research over the past 30 years combining the approaches to investigate the way faces communicate social information. Below, these applications are considered in more detail.

\section{The 'Method of Extremes'}

One extremely common use of computer graphics techniques in face perception research is the use of facial averaging to represent the prototypical appearance of individuals that score high or low (i.e, extreme) on a particular trait or social measure. This hypothesis driven approach (Sutherland, Rhodes, \& Young, 2017) is based on the premise that traits that differ between individuals, and are related to facial appearance, will be clearer in these average faces - that is, the average faces of those scoring high or low on a trait will appear different, but only if that trait covaries with facial appearance (Perrett, May, \& Yoshikawa, 1994).

Otherwise, the faces will tend toward the average appearance. This method has successfully revealed many intriguing findings in social perception. For example, people high or low on personality traits like Extraversion, Neuroticism, or 
Agreeableness seem to have distinct facial appearances (Jones, Kramer, \& Ward, 2012; Kramer \& Ward, 2010; Little \& Perrett, 2007; Penton-Voak, Pound, Little, \& Perrett, 2006), as do those scoring high or low on the Dark Triad (Holtzman, 2011). Individuals with high or low sociosexuality also possess distinct facial appearances that observers are able to discriminate between (Boothroyd, Jones, Burt, DeBruine, \& Perrett, 2008). The method of extremes has also been employed to uncover surprising relationships between appearances on measures such as reaction time variability, with individuals with faster and more consistent reaction times possessing more attractive faces on average (Butler, Saville, Ward, \& Ramsey, 2017).

The traits that are entered into composites need not be individual differences related to the comprising faces. In fact, the most common application of this method is to utilise perceptual measures and calculate the prototypical face of individuals who appear high or low on a social dimension. This been extensively applied in studying attractiveness, where composite faces of those perceived high and low in attractiveness reveal key differences in the properties of what observers rate as attractive (DeBruine, Jones, Unger, Little, \& Feinberg, 2007). Apparent perceived health composites have also been used to assess preferences under certain situations (Smith, Jones, DeBruine, \& Little, 2009), or lead to more data-driven explorations of differences between those who appear high or low in health (Jones, Porcheron, Sweda, Morizot, \& Russell, 2016). Importantly, key models of social perception have been developed around the perceived appearance of individuals who are high or low on the evaluative dimensions of attractiveness, trustworthiness, and dominance (Sutherland et al., 2013) using this approach.

\section{The 'method of extremes' and transforms}


Despite uncovering intriguing differences between populations of people, composites suffer from rather poor ecological validity - they do not look like real faces, as they possess qualities typical of averaged images, such as smooth skin texture. Rather than present composite images as stimuli to test psychological hypotheses, individual faces can be warped along the continuum defined by a pair of anchor faces, blending a target face between the appearances of the anchors effectively increasing or decreasing the information present in the anchor faces in an actual face. This technique has been very widely used across many areas of research. Popular applications include morphing faces between the average faces of many females and males to induce a sexual dimorphism transform to individual faces, to test the relative preferences of observers for these facial qualities (Glassenberg, Feinberg, Jones, Little, \& DeBruine, 2010; Perrett et al., 1994). Other applications involve warping faces between individuals with relatively more or less symptoms of depression (Scott, Kramer, Jones, \& Ward, 2013), transforming faces between average and very attractive appearances to alter their distinctiveness or typicality (Sofer, Dotsch, Wigboldus, \& Todorov, 2015), manipulating apparent cues to height in faces (Re, DeBruine, Jones, \& Perrett, 2013), increasing or decreasing qualities associated with perceived health (Re, DeBruine, Jones, \& Perrett, 2013), altering the appearance of faces perceived as high or low on the Big 5 (Sutherland et al., 2015), and manipulating perceived trustworthiness and dominance to examine how clinical samples respond to these cues (Sprengelmeyer et al., 2016). This approach producing composite images, and using them to warp individual faces between the composites in a gradated fashion - has recently been referred to data-driven, as it requires sampling a range of faces to create the composites before applying the 
changes across a subset of individual faces (Sutherland et al., 2017), and allows testing of observations that emerge from composite faces.

\section{Criticisms of the 'method of extremes'}

The above sections have made clear how prevalent the general methodology facial averaging and transforms - is in studying social perception with faces. However, to date, this general approach has received almost no criticism within the literature. This section outlines several interrelated theoretical issues that are fundamentally important to applying this general methodology to social perception, and existing approaches that might alleviate these concerns.

1) The method of extremes and transforms are not data driven. Recent reviews have characterised the general methodology - creating composites and implementing them in face warping - as a data driven approach to studying social perception (Sutherland et al., 2017). Using the general methodology, a large sample of faces is gathered, rated or measured for a social trait, and composites of those scoring high and low scoring are created. If the composites appear sufficiently different, indicating covariation between appearance and the trait, individual faces can then be warped between the composites, creating appearances manipulated along the putative dimension. This provides the defined data-driven method that combines with hypothesis testing - how do observers react to these warped images, assuming the manipulated appearance is linked with the differences between the composites?

The first issue with the general methodology is that it does not lend itself well to being data-driven. While a large sample of faces is necessary to create the composites, the composites themselves are often based on a fraction of the observed data. A commonly observed value in the literature is using between 15 and 20 faces to create the high and low composites (though this is less common in studies examining 
sexual dimorphism, where high numbers of females and males are incorporated into the averages; Jones et al., 2018). Some examples are highlighted in Table 1, illustrating the main point: large percentages of the data are actually thrown away when creating composites. The result is that composites and face warping, which are widely relied upon, are based inherently on the average appearance of a small number of individuals. This does not fit the criteria for a data-driven methodology. The discarding of large parts of a dataset to produce composites results in another issue: given the small sample of faces that comprise the composites, it is possible that a number of them are extreme outliers. In the same way a statistical point estimate score with a small sample is heavily influenced by outliers, a select few individuals may cause the differences observed in composite faces.

Table 1. Examples of the method of extremes in the face perception literature

\begin{tabular}{ccccc}
\hline Study & $\begin{array}{c}\text { Number of faces } \\
\text { (Female, Male) }\end{array}$ & $\begin{array}{c}\text { Faces used in } \\
\text { composites } \\
\text { (per sex) }\end{array}$ & $\begin{array}{c}\text { Number of } \\
\text { composite } \\
\text { pairs (per sex) }\end{array}$ & $\begin{array}{c}\text { Utilises } \\
\text { transforms }\end{array}$ \\
\hline Little \& Perrett (2007) & 123,68 & 15 & 5 & $\mathrm{~N}$ \\
Jones et al (2016) & $146,-$ & 12 & 1 & $\mathrm{~N}$ \\
Jones et al (2012) & $92,-$ & 15 & 5 & $\mathrm{~N}$ \\
Kramer \& Ward (2010) & $63,-$ & 15 & 7 & $\mathrm{~N}$ \\
Oldmeadow et al & 250,250 & 20 & 4 & $\mathrm{~N}$ \\
(2012) & & & 5 & $\mathrm{Y}$ \\
Penton-Voak et al & 148,146 & 15 & 1 & $\mathrm{Y}$ \\
(2006) & 83,47 & 10 & 1 & $\mathrm{Y}$ \\
Re et al (2013) & 130,95 & 15 & 1 & $\mathrm{Y}$ \\
Scott et al (2013) & 92, & 92,12 & 7 & $\mathrm{Y}$ \\
Sofer et al (2013) & 500,500 & 20 & 5 & $\mathrm{Y}$ \\
Sutherland et al (2013) & 500,500 & 20 & & \\
Sutherland et al (2015) & & & & \\
\hline
\end{tabular}


Researchers have made bounds in using more genuinely data driven approaches to understanding social perception. Principal Components Analysis (PCA) has been a fruitful avenue in this regard. By projecting high dimensional face data into a lower dimensional space, where each face is represented as an $n$-dimensional vector or projection, researchers can define axes in this space and compute the vector projection of where each face lies along this axis. This has been used successfully to produce image transforms based on the full range of data for a range of attributes, such as facial masculinity (Holzleitner et al., 2014; Holzleitner \& Perrett, 2016) or body mass index (Henderson, Holzleitner, Talamas, \& Perrett, 2016). Indeed, with this method, it is possible to select individuals who are facially objectively low or high on a trait, even if they are not rated as the highest or lowest in a dataset. One drawback of this method is that the initial axes within the face-space are computed by averaging the vector positions of individuals high or low on a given trait, to define a start and end point of the axes. While all the data is used, faces are again scored relative to a small number of extreme scorers for a given trait (Henderson et al., 2016).

Similar methods have used complex vector projections in face space to reveal the characteristic appearance of faces perceived as high or low on perceived social traits (Adolphs, Nummenmaa, Todorov, \& Haxby, 2016; Oosterhof \& Todorov, 2008; Todorov, Dotsch, Porter, Oosterhof, \& Falvello, 2013). These methods offer a powerful alternative approach to averaging and warping as they use all the available data and do not rely on point estimates from average faces. However, they are widely validated on synthetic three dimensional faces, where shape information is incredibly rich (several thousand 3D vertices as compared to hundreds of $x, y$ landmark points), and components are typically discarded as part of the analysis (Oosterhof \& Todorov, 
2008), an approach which may not translate well to photographs of faces, which are more widely used. They may also not reflect how these judgments occur with real faces, as key judgments like trustworthiness are impaired using synthetic faces (Balas $\&$ Pacella, 2017). Additionally, these vector techniques are not easily accessible to most researchers, and are not simply interpreted in the way a face transform is (e.g., $20 \%$ of the difference between face A and B, applied to face C).

Research that has adapted shape analysis methods from comparative biology, in particular geometric morphometrics (GMM), has also made progress into more data-driven understandings of social perception. Using multivariate regression to predict facial shape, GMM utilises all available shape data to predict faces of individuals along the scale of traits like perceived and actual intelligence (Kleisner, Chvátalová, \& Flegr, 2014), and has shown how shapes associated with perceived trustworthiness, with attractiveness controlled, contribute to real life outcomes such as position within corporate hierarchies (Linke, Saribay, \& Kleisner, 2016). These findings would be almost impossible to find with the method of extremes. While thus far focusing exclusively on shape, these methods recently been applied to examine variation in facial texture (Mayer, Windhager, Schaefer, \& Mitteroecker, 2017), which is an extensive source of social information. Unfortunately, these approaches have seen little uptake with the face perception literature, possibly due to their mathematical complexity and specialist software packages, such as the TPS suite (Rohlf, 2015).

\section{2) The method of extremes captures more variation than is of interest.}

The above section highlighted that the method of extremes combined with transforms is not strictly data driven, and generates stimuli that are based on small numbers of individuals. Another important theoretical consideration that is related to the above 
point is inspired by ecological approaches to psychology (Brunswik, 1956). In the real world, information content is correlated, especially in the context of impressions of high dimensional stimuli like faces (Secord, 1958). Yet, the vast majority of face research is reductionist in its approach, using warping and averaging to examine single cues in isolation (Penton-Voak, 2011).

Given this assumption, then by design the method of extremes cannot examine a single cue in isolation, which is the general approach it is used for. As an example, consider the ubiquity of the correlation of trustworthiness with almost any other social judgment (Todorov, Said, Engell, \& Oosterhof, 2008). Recent reviews characterise averaging faces perceived as high or low trustworthiness as a way for researchers to visualise and manipulate the cues associated with perceived trustworthiness (Sutherland et al., 2017). While this is surely the case, given that information content is correlated, there is little chance cues solely related to trustworthiness are being visualised. In fact, the composites are likely to produce the appearance of people who are also perceived as low in dominance, a common negative correlation with trustworthiness (Todorov et al., 2008), or who are rated as attractive, which is generally positively correlated (Fruhen, Watkins, \& Jones, 2015). Indeed, controlling attractiveness in facial shape reveals new information when studying trustworthiness (Linke et al., 2016). If trustworthiness composites are used to transform images with the intention of manipulating apparent trust, a researcher is also manipulating cooccurring cues and not trustworthiness alone, with all the theoretical implications this may have.

There are several examples of this occurring in the literature. Perceived Big 5 composites are generated from highly correlated ratings (up to .85; Sutherland et al., 2013), group stereotypes emerge from correlated ratings (Oldmeadow, Sutherland, \& 
Young, 2013), as well as intelligence and attractiveness (Sutherland et al., 2013), manipulated dominance (Bird et al., 2017), or both attractiveness and dominance (Little, Roberts, Jones, \& DeBruine, 2012). Even if a study does not collect or report additional trait ratings for those images going into the composites, there is sufficient evidence to suggest that almost any trait is correlated with another, in line with predictions of ecological psychology (Brunswik, 1956). More generally, what goes into composite faces and is manipulated is confounded with other traits. Indeed, given the correlations of these traits, it is likely that individual faces with extreme scores on one trait (for example, trustworthiness) may also be entered into the composite of another correlated trait (e.g., attractiveness), basing yet more assumptions on a small set of extreme appearances.

This issue has not gone unnoticed by researchers. Steps taken to alleviate this confound have included extensive permutations of the extreme-scoring individuals to produce many high and low composites based on smaller numbers of individuals (Scott, Jones, Kramer, \& Ward, 2015), or using regression to control for the influence of other traits, then sampling the extremes from the residual scorers (Little et al., 2012). Others have implemented neural networks to create weighted appearances of single traits while controlling for others (Vernon, Sutherland, Young, \& Hartley, 2014). Face-space vector methods are able to produce orthogonal axes of variation with perceived traits (Todorov et al., 2013), producing faces that vary on a single trait, with influences of other traits controlled. However, these methods have drawbacks in that they either throw away large amounts of the data used to generate the composites, or are based on synthetic faces.

\section{3) The method of extremes and transforms assume a linear relationship.}

The final theoretical issue involved in the general methodology is that is makes a 
fundamental assumption that variation between a trait and facial appearance is linear. That is, to move from the average appearance of individuals high in a perceived or actual trait to those low in the same trait involves a linear change in facial appearance. The reasoning for this assumption arises simply from the way in which image warping is implemented. As outlined above, each face is divided into a set of triangles, and each of these triangles is affine warped to the corresponding triangle in another image. This affine warp is linear in nature, capable of retaining straight lines and distance ratios. As such, any transforms between faces are a linear result.

The standard approach would be to warp faces between composites, which may reveal a linear change in perception according to the amount of the transform (e.g., Sutherland et al., 2015). However, this is not a convincing demonstration that these perceptions are linear in the real world, given that the composites are based on small samples inherently extreme scoring faces that are likely conflated with other extreme kinds of cues. To say that facial appearance covaries linearly based on these transforms is then circular - a small number of extreme faces are averaged, and linear differences applied to individual faces. While the resulting perceptions may change linearly, it is not clear whether this works in reality, and is at best a proxy score for how social perception with faces may work. Indeed, research using data-driven multivariate regression on just facial shape has found that genuine linear changes in appearance can involve non-linear responses by observers, further calling into question the utility of warping images (Windhager et al., 2018).

However, there is evidence that facial appearance and traits do vary in a linear fashion, though not within any easily interpretable statistical framework. For example, neural networks with linearly activated layers achieve reasonable accuracy in predicting social traits (Vernon et al., 2014) when given hypothesis-driven shape 
attributes (such as mouth width and height, indexing expressions like smiles), without accounting for texture information. However, other evidence shows simple properties like body mass index (BMI) in faces are better explained by non-linear approaches that utilise both shape and texture information, particularly through the use of support vector machines (Wolffhechel et al., 2015). The evidence that social trait perception and facial appearance covary linearly is therefore limited to complex statistical approaches, and is not convincingly demonstrated by the method of extremes.

To summarise, the three theoretical concerns with the general methodology in much face perception research is that composites are based on a small subsample of a larger dataset, which is wasteful. Second, given the demonstration that almost all social traits are correlated, composites of faces and warps between them are likely confounded with other traits. Third, the assumption that trait perceptions change linearly in response to the warped appearance between composites may not be reflective of the nature of real world perception and appearance. While there exists ways around these three issues, no one method is simultaneously accessible, interpretable, and ecologically valid. The next section outlines a novel approach to examining common questions in face perception, and introduces a dedicated computer software package to do so.

\section{Introducing Face Regression}

A solution to the above theoretical concerns is an approach termed here as face regression. Suppose a researcher wishes to examine how facial appearance might covary with either a single perceived trait, or a set of traits. One solution is to decompose a sample of faces with PCA, and use this lower dimensional space to predict the perceived outcome measure. Components, corresponding to aspects of facial appearance that best predict the outcome measure, can then be recovered. 
However, predicting more than one trait becomes more difficult, and collecting many trait ratings of faces is common practice. How to simultaneously model the elements of appearance that are related to certain perceived traits? One solution is to reverse the direction of prediction by using the trait ratings or scores themselves to predict elements of appearance. This has been employed on shape information alone by GMM researchers (Kleisner et al., 2014; Linke et al., 2016), but has been used rarely to examine all factors of appearance in an accessible way (Mayer et al., 2017; Burt et al., 2007), but has not been discussed or used elsewhere.

The face regression approach utilises an algorithm that maps a regression formula to every dimension of a set of faces. The predictor variables (or features) for each regression are the set of social trait ratings (e.g. perceived attractiveness, trustworthiness) or qualities (e.g. BMI, sex, actual health measures) associated with each face. The dependent variables (that is, the dimensions of faces) are the shape coordinates (in two or three dimensions) or colour pixel values (in whichever colour space the face textures are represented in, e.g. RGB or CIEL*a*b*). This modelling approach produces a regression formula for each shape coordinate, and for each pixel in each channel of a given colour space. These formulas contain weights for each predictor, indicating the importance of that particular element for a given trait, stored in a multidimensional array.

With these formulas, a completely data-driven approach is achieved: a value for each trait can be passed through the formula array, and every single aspect of the resulting face is adjusted based on parameters calculated from the entire image set. This approach is also easily interpretable to anyone familiar with regression, and while mathematically similar to the multivariate regressions used elsewhere (Linke et al., 2016), requires little knowledge beyond basic statistics. A prediction can be made 
by passing a value for each weight into a given regression formula - here, the prediction is a facial appearance, and a value is passed to a large array of weights that map all shape and colour features of faces.

Furthermore, this approach offers great flexibility, in that values for each predictor variable can be passed separately and simultaneously, holding constant the variance for specific predictors, effectively removing their presence. Alternatively, contrasting appearances can be generated that would be difficult to visualise given the direction of correlations in the real world, by passing low and high numbers to certain predictors. A general layout of the approach is illustrated in Figure 1, where a visual depiction of the algorithm is presented.

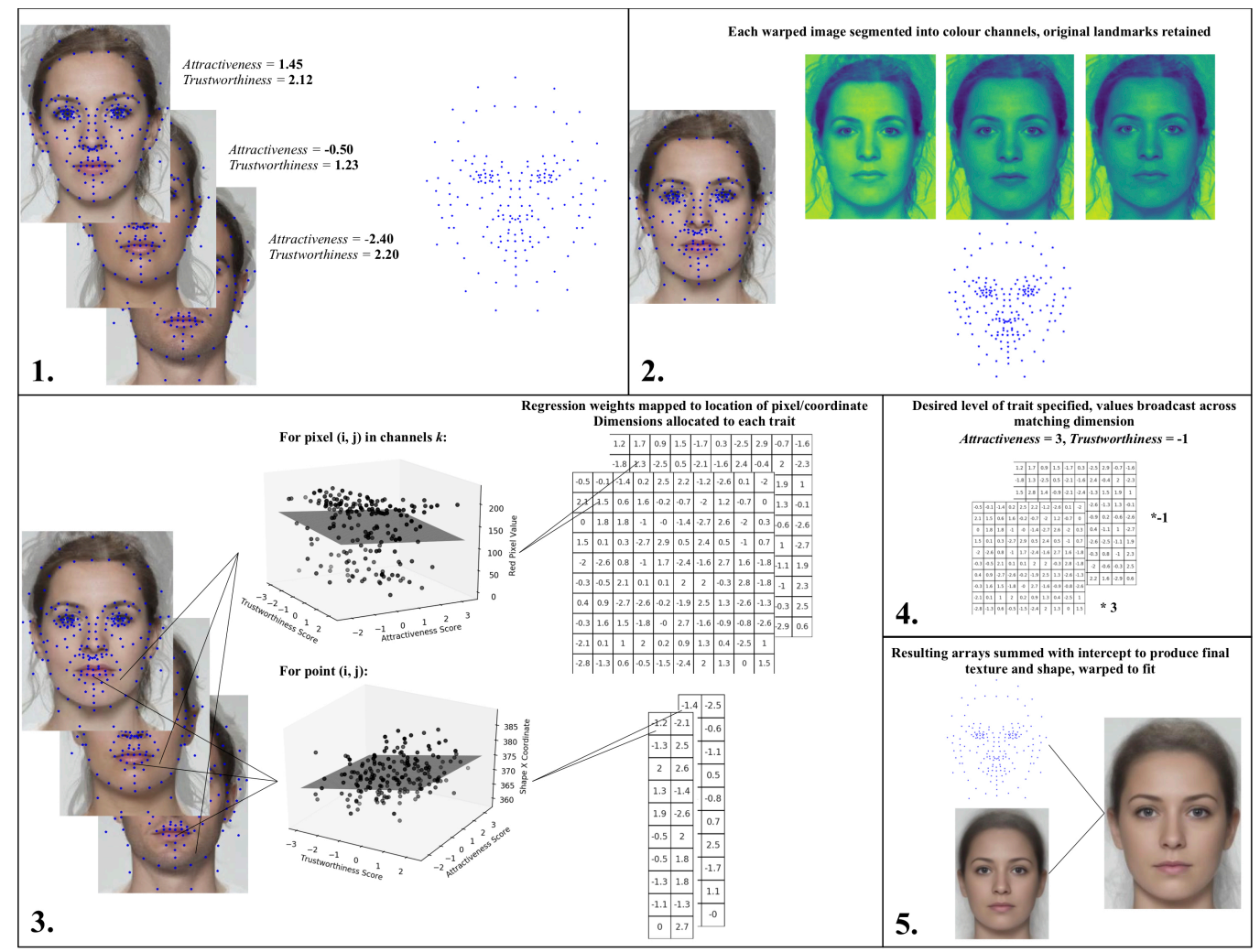

Figure 1. Visual depiction of the face regression algorithm, modelling the traits Trustworthiness and Attractiveness. In 1, the average face shape is computed for a set of faces with known values on the traits. In 2, each face is warped to the average face shape, each colour channel is extracted, and the original landmarks are retained. In 3, each element (every single pixel and coordinate point) is extracted from all faces and a linear model is built using the trait scores for each face. The resulting 
weights are stored; building a multidimensional array that contains a formula representing the individual dimension (coordinate, pixel). In 4, a desired value for traits are passed by to the array, which is broadcast across the matching dimension, providing the model's best guess to the appearance of that face. In 5 , the predicted texture is warped from the average face shape to the predicted face shape to produce the final outcome.

\section{Face Regression Algorithm and Implementation}

The general approach to the modelling algorithm is described here. For a set of faces $N$, measured on a set of traits $M$, with associated shape coordinates $P$ in $Y$ dimensions, and a face texture image of the same height and width across all faces $Q$, in three dimensions, the algorithm proceeds as follows:

a) The average face shape is computed by computing the mean across all points $P$.

b) Each face image $Q$ is warped using Delaunay triangulation and affine warps with bilinear pixel interpolation to the average appearance, and each colour channel is separated. These standardised arrays $\left(Q_{\mathrm{St}}^{1} Q_{\mathrm{St}}^{2}, Q_{\mathrm{St}}^{3}\right)$ represent the facial-shape free colour channels for each member of $N$.

c) For element $i, j$ (coordinate point) in $P$, a regression formula with $M$ traits is computed against the same element extracted from all faces. Each regression weight is stored in an array of size $P Y M$.

d) The above step is repeated for each array $Q_{\mathrm{St}}^{1} Q_{\mathrm{St}}^{2} Q_{\mathrm{st}}^{3}$, creating arrays for each colour channel of regression weights for each pixel, yielding arrays $Q_{\mathrm{St}}^{1} M, Q_{\mathrm{St}}^{2}$ $M, Q_{\mathrm{St}}^{3} M$.

e) A desired value for each trait in $M$ is passed to the shape and colour arrays, now containing regression weights, and the value is broadcast across the individual weights in the matching dimension. The resulting colour arrays are recombined into an image with the same input dimensions as $Q$. 
f) The combined colour array undergoes warping by the same procedure as step 2 from the average shape, $P$, to the predicted shape obtained from PYM.

Given that the above algorithm uses no data reduction, it can be slow for large images. However, it is implemented in a well-optimised Python package - the Python Face Regressor (PyFaceR; https://github.com/alexjonesphd/python-face-regressor) with a simple and easy to use application programming interface, that allows users to standardise the size of a set of landmarked images, warp each image to the average shape and fit the regression weights, and make predictions and save images and associated landmark files with a small set of intuitive commands. The package is built around the powerful data science tools implemented in Python, and also contains an interactive visualisation tool built on Bokeh and Jupyter notebooks. Examples are available at the Open Science Framework (osf.io/q5wvn) with notebooks demonstrating the analyses carried out here, serving as a user guide. It is worth noting that the algorithm is overly cautious - it is likely that regions of faces likely share very similar regression weights (e.g., it is unlikely each pixel of the eyes varies drastically with a given trait), and so some assumptions could be made. However, given the current speed of computation and Python implementation of the algorithm, the extra steps are negligible, and one of the primary aims of this approach is to be truly data driven.

\section{Applying Face Regression - Study One}

In this first example, the algorithm is applied to and validated against a common topic in the literature: facial attractiveness. Attractiveness is an exceptionally well studied aspect of face perception (Little, Jones, \& DeBruine, 2011; Rhodes, 2006), with some evidence that as appearance deviates from the average, 
attractiveness ratings decrease linearly (Deffenbacher, Vetter, Johanson, \& O’Toole, 1998). However, significant proportion of work studying attractiveness has relied on the method of extremes and transforming (Little et al., 2011; Rhodes, 2006), using faces at extreme ends of the scale. Here, the face regression approach is pitted against the method of extremes, to examine if attractiveness ratings scale linearly with appearance, and how well the face regression approach can capture the variance in appearance that facial averaging does.

\section{Method}

The data set utilised here is the open-access Face Research Lab London Set (DeBruine \& Jones, 2017), comprising 102 faces (49 females, age $M=27.72, S D=$ 7.11 , range $=18-54)$, rated for attractiveness by 2513 observers (1552 females, 3 intersex, $M=26.72, S D=10.04$, range $=17-88$ ). Faces were rated for attractiveness on a one (low) to seven (high) scale. Each face is delineated with a series of 189 landmarks, describing the shape properties of the face by outlining the fiducial points, and photographed against a plain white background with a neutral expression, with hair held back from the face. Images were resized from 1350 x 1350 to 300 x 500 using PyFaceR. While modelling a range of responses in this dataset is possible, here only ratings made by heterosexual individuals of the opposite sex faces (i.e. females rating male faces) were incorporated. This yielded a final sample of 1096 female and 664 male raters, and allows the application of the algorithm to the perceptions of heterosexual participants judging facial attractiveness.

First, using the method of extremes, the 15 highest and lowest rated females (as rated by males) and the 15 highest and lowest males (as rated by females) were averaged. These are shown in Figure 2, along with their associated mean attractiveness rating - that is, the average attractiveness rating assigned to these 
extreme-scoring faces. It is apparent that the method produces composite faces that vary in attractiveness, but also highlights some of the limitations of the method. First, the method does not always capture an ideal amount of variation. The high attractiveness composites sample from around the midpoint of the scale, and the mean attractiveness of the faces is generally not far removed from the midpoint of the scale - between three and four, out of seven. As can also be seen from the distributions, only a few highly attractive faces are included, while many mid-attractiveness faces are retained.

The data in Figure 2 provide estimates that can be used with the face regression algorithm. A simple prediction is that if attractiveness and facial appearance covary linearly, then face regression should be able to recreate the composite images by using the mean attractiveness score of the faces that comprise the composite. However, these faces would be generated from all available data, and not just based on the extreme scorers. To achieve this, two models were built - one for female faces as rated by male observers, and one for male faces rated by female observers. The mean rating of attractiveness for each face was used as a predictor variable to predict every element of shape coordinates and pixel values in the RGB colour space. These 'regressed' faces are shown in Figure 3. 

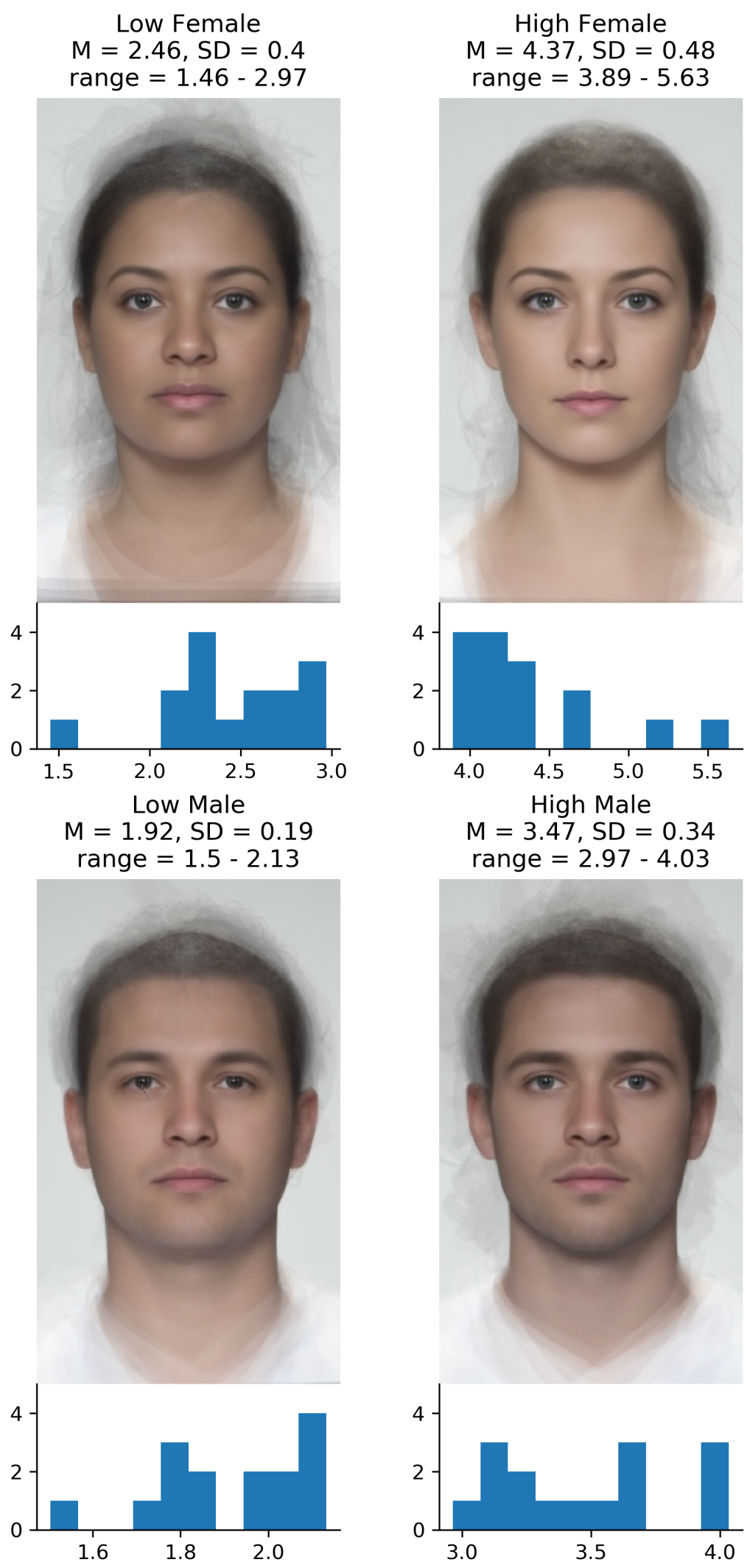

Figure 2. The method of extremes applied to the highest and lowest 15 scorers in attractiveness in the London face set. Descriptive statistics of the faces that comprise the composites are provided, and histograms indicate the general spread of scores and counts of individuals entered into the composite. 


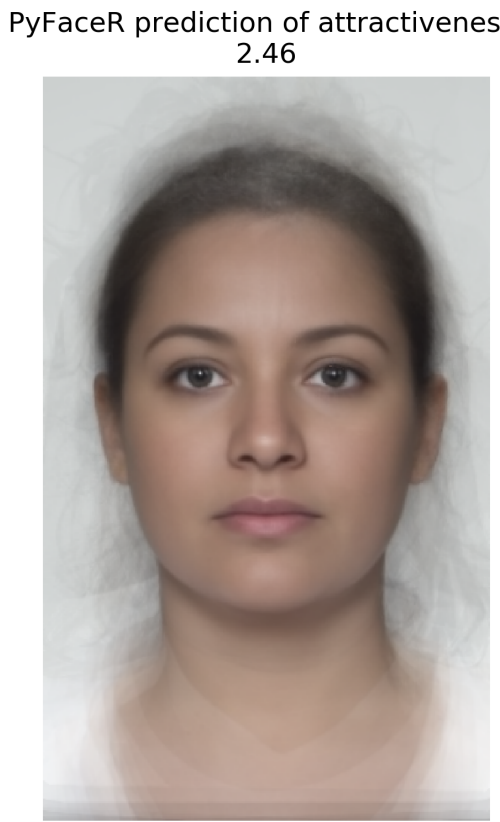

PyFaceR prediction of attractiveness: 1.92

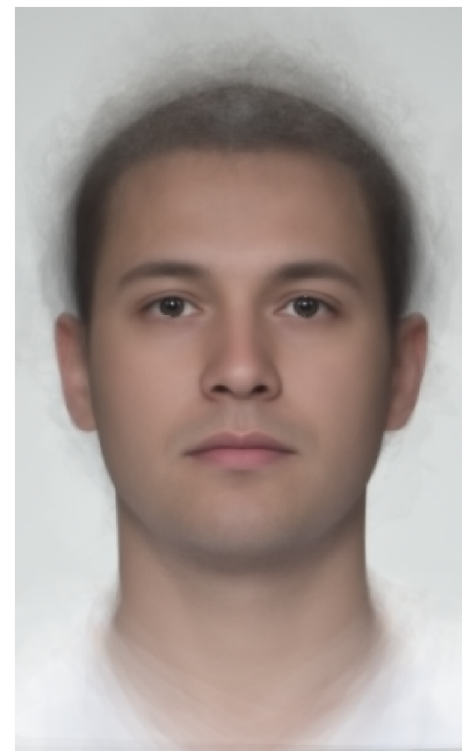

PyFaceR prediction of attractiveness: 4.37

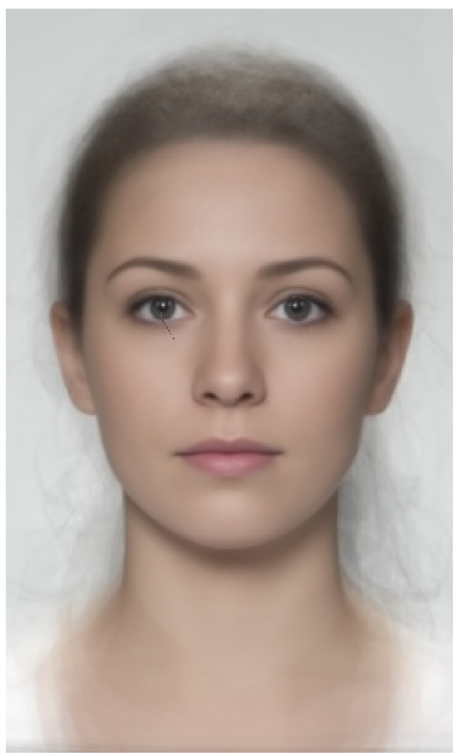

PyFaceR prediction of attractiveness: 3.47

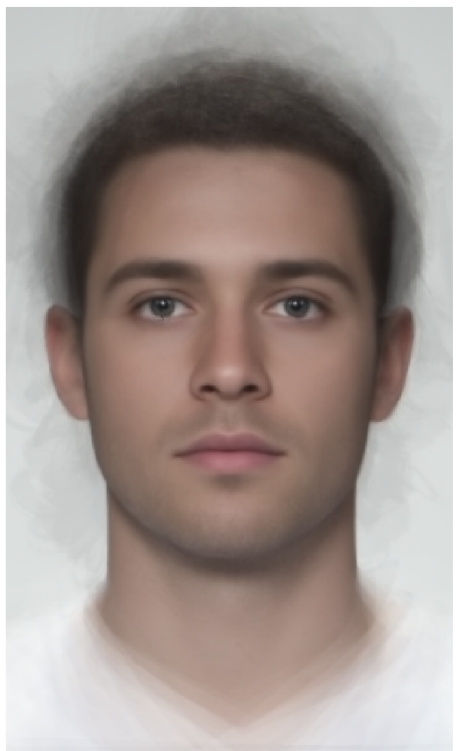

Figure 3. The face regression technique applied to the whole dataset. The mean ratings of the faces that comprised the composites in Figure 2 were entered into the model, which mapped regression weights to every aspect of shape and texture in the dataset using the attractiveness ratings of each face.

The faces in Figure 3 are a convincing demonstration of the utility of the method, given that the regressed faces are almost identical to those produced by the method of extremes. There are two points worth making here. First, it demonstrates 
that the method of extremes effectively captures the variance in facial appearance at a given point in the perceptual rating scale. Second, it confirms that every aspect of facial appearance varies linearly with perceived attractiveness. Rather than rely on inferences from transforms, the regression approach confirms intermediate faces also vary linearly.

So far, the method of extremes and face regression are able to produce virtually identical outcomes. One of the advantages the face regression approach has in this single-predictor scenario is its interpretability - the predicted faces merely represent the appearance of a face in a given position along the rating scale used in the study, rather than a composite of the lowest or highest rated faces. To further illustrate the flexibility of the approach, simple values on the rating scale can be passed to the model to predict the appearance of faces rated low or high, respectively. However, given that the approach is based on regression, it suffers the same caveats namely, extrapolating outside the range of available data will lead to unstable prediction errors (Snee, 1977). In Figure 4, the model predicts the appearance of faces with low and high values in the rating scale - two and six. The distortions in the image are the result of textures being clipped to stay within the bounds of pixel ranges (0-255), which the regressions can predict outside of, and effectively visualise the extent of moving outside of the data range: no face received an average score of one or seven, and more extreme clipping in the male faces reflects their more restricted range of attractiveness scores. As such, two and six represent general limits of attractiveness ratings. As a way to further demonstrate the linear relationship between attractiveness and facial appearance, consider the additional faces to the right of Figure 4, generated using the method of extremes. The high and low perceived attractiveness composites are used as anchor points, and then the average of all sex- 
matched faces are caricatured (warped beyond the high attractiveness composite), by multiplying the linear difference between the composites by a weighting of 1.4 . The overall appearance is again strikingly similar, confirming the linear relationship between appearance and attractiveness, but the transform value is less interpretable.

PyFaceR prediction of attractiveness: 2

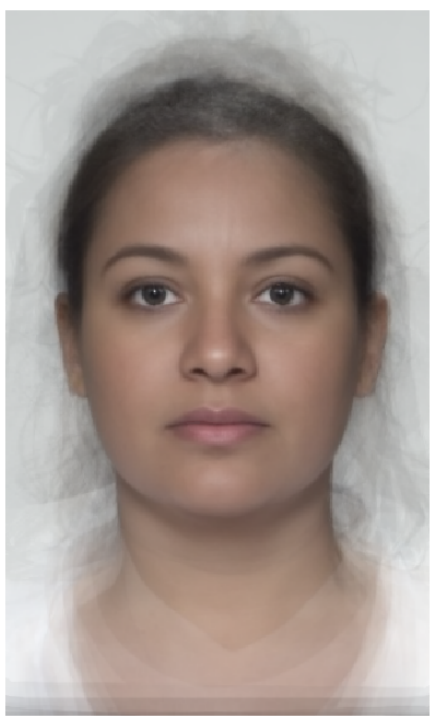

PyFaceR prediction of attractiveness: 2

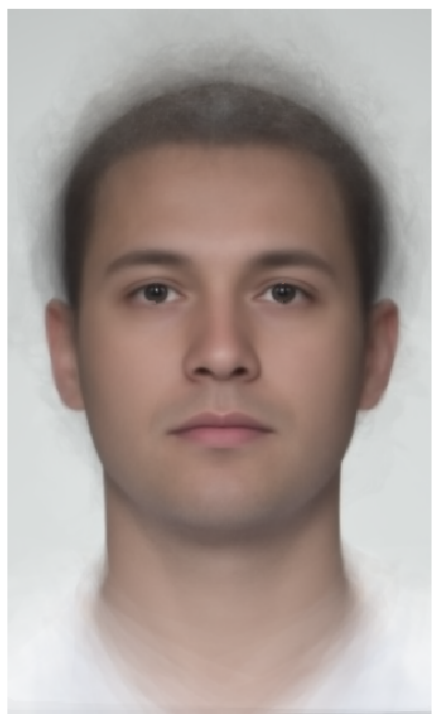

PyFaceR prediction

of attractiveness:

6

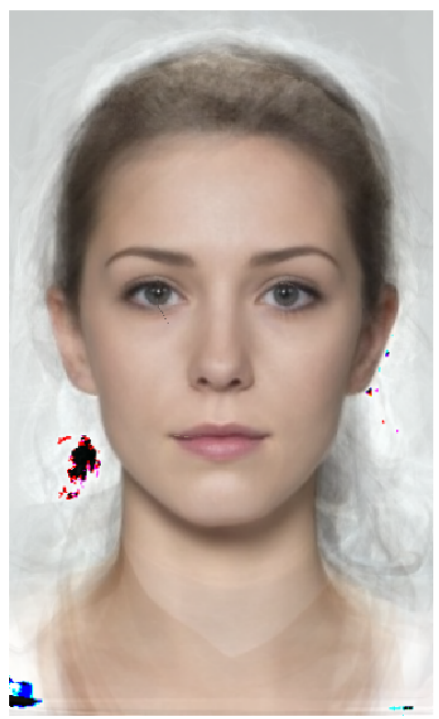

PyFaceR prediction of attractiveness: 6

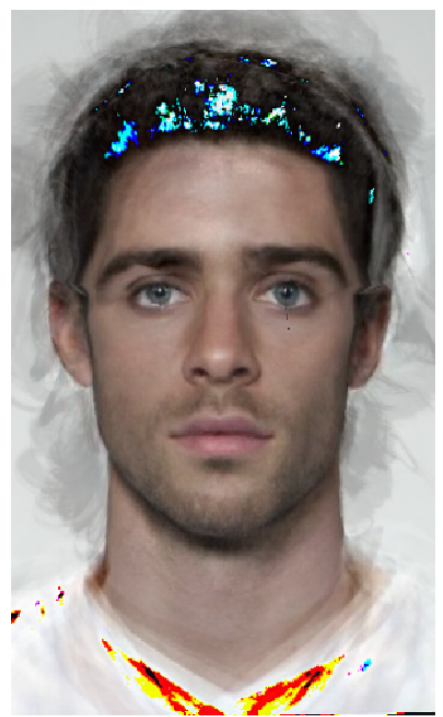

Caricatured average face

towards high

attractive composite

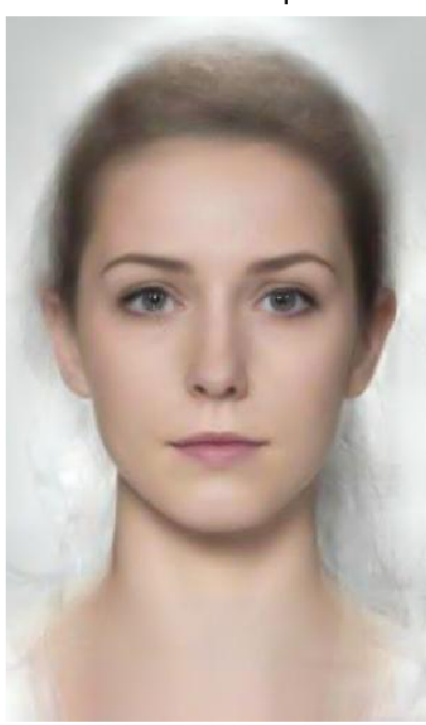

Caricatured average face towards high attractive composite

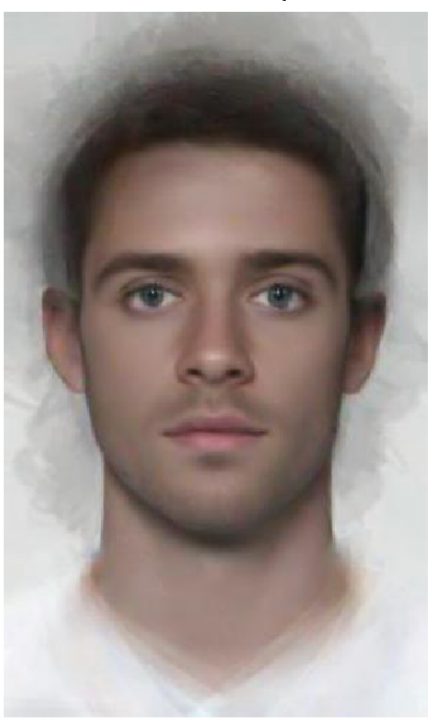

Figure 4. Illustration of using face regression to predict values along the rating scale used in the study.

The algorithm captures the low and high appearances successfully, but there is evidence of certain areas outside of the face being clipped, indicating a prediction beyond the permitted integer range for 
images. Images on the right indicate the method of extremes being used to transform the average facial shape of either sex past the high attractiveness composite, again confirming the linear relationship between appearance and attractiveness.

This initial study has benchmarked the face regression algorithm against the method of extremes to illustrate several theoretically important points. First, it can be seen that the method of extremes is effective in capturing the covariance of attractiveness and facial appearance in a linear fashion - the composites are effective 'snapshots' of appearance at the mean attractiveness score they compromise, confirmed by the direct linear relationship captured by the face regression algorithm. Second, it has shown the relative interpretability of the face regression approach compared to the method of extremes in relation to the dataset. Caricaturing a face by an arbitrary amount produced very similar appearances to the algorithm predicting an appearance at high or low points on the scale. More generally, this study has confirmed the linear relationship long assumed by the method of extremes.

This study used a single predictor, perceived attractiveness, to demonstrate essentially similar functionality to the method of extremes, despite being fully data driven in comparison. In the next study, the real strengths of the algorithm are leveraged, by taking advantage of the statistical properties of multiple regression, with functionality that is impossible with the method of extremes.

\section{Applying Face Regression - Study Two}

The purpose of this study is to demonstrate how the face regression algorithm can isolate a single trait in faces, while holding others constant. One of the main assumptions of the method of extremes is that the composite presents the appearance of a single trait, but this is very unlikely to be achieved given the correlated nature of perception and information content in the real world (Brunswik, 1956). Here, the face 
regression approach is compared against the method of extremes, and differences in the perceptions of observers are carried out.

\section{Method}

For this study, the Oslo Face Database (Chelnokova et al., 2014) was utilised. The database comprises photographs of 210 young adults (116 females). Models were photographed against a standard background with a neutral facial expression. The dataset contains three gaze profiles, but for this study only the direct gaze images were utilised. The dataset contains ratings for the three factor model of social perception (Sutherland et al., 2013) - Attractiveness, Dominance, and Trustworthiness. Each trait was rated on a visual analogue scale by a separate sample of raters (Attractiveness: $N=40,21$ females, age $M=26.00, S D=7.50$; Dominance: $N=20$, all males, age $M=25.00, S D=4.30$; Trustworthiness: $N=40,20$ females, age $M=26.70, S D=6.50$ ), and average scores were computed across raters for each face from the raw data provided. A subset of faces (14 females, 10 males) was not rated for either trustworthiness or dominance, or both. Discarding their data led to a final sample of 186 faces (102 females) that were modelled.

All faces were delineated automatically using the facial landmark detector in the Python implementation of the Dlib machine learning package (King, 2009). The detector uses the histogram of gradient descent method to detect landmarks, trained on a set of 300 naturalistic faces (Sagonas, Antonakos, Tzimiropoulos, Zafeiriou, \& Pantic, 2016) to produce a set of 68 landmarks outlining the major facial features such as the eyebrows, eyes, nose, mouth, and jawline.

\section{Analysis}


After averaging data across observers to provide a mean score for each face, pairwise correlations were conducted for each possible variable combination. This was conducted separately for each sex. These correlations are displayed in Figure 5.
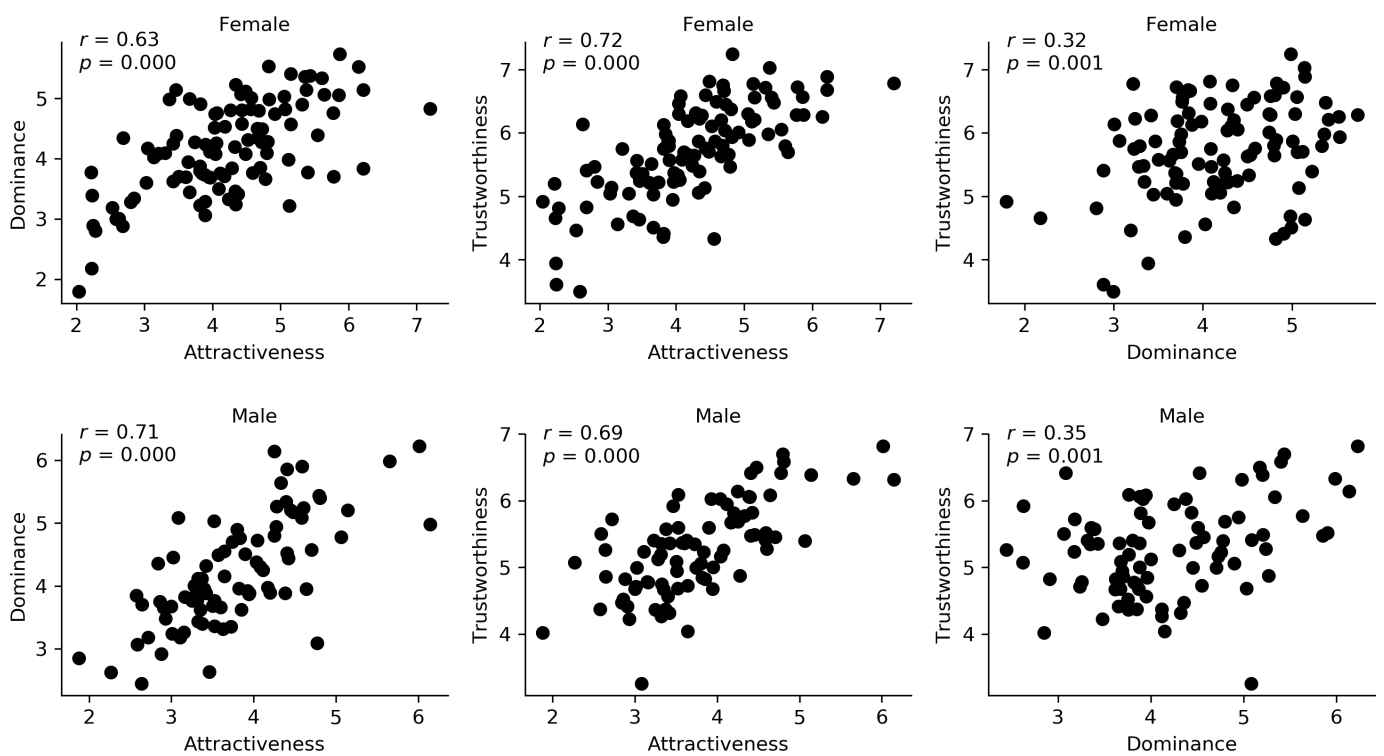

Figure 5. Pairwise Pearson correlations between ratings of social traits, conducted for both female and male faces separately. Spearman rank correlations yielded identical results within 0.04 of the parametric equivalent.

Figure 5 demonstrates the second theoretical point raised in the introduction in reality, perceptions are correlated, and taking averages of faces at the low and high end of the scale will capture variance in other traits, measured or otherwise, and that this variance may influence the perceptions of observers. In the case of a multivariate study, where composites may be generated for multiple variables, these correlations may also lead to significant overlap in the individuals who enter into the composites across traits. More simply, people who appear extreme on one trait are likely to appear extreme on the other, leading to overlap in the appearances of the composites.

To test this, a simple count was taken of the number of times the same individuals in the high and low attractiveness composites for both sexes appeared in the high and low trustworthiness and dominance composites, or both, using the 15 
most extreme scorers. These counts are displayed in Table 2. The $p$-value of observing each of these counts was computed through bootstrap resampling. The 15 high and low scorers for female and male attractiveness were taken, but for the traits trustworthiness and dominance, the high and low 15 scorers were chosen randomly without replacement. The degree of overlap was then computed for trustworthiness, dominance, and both composites, and repeated 5000 times. The $p$-values were then computed from the generated samples - that is, given the generated sample, what is the probability of observing an overlap equal to or greater than what was observed in the initial data?

Table 2. Count data and $p$ values of the 15 faces in high/low attractiveness composite appearing in another composite, or both

\begin{tabular}{ccccc}
\hline & \multicolumn{2}{c}{ Female } & \multicolumn{2}{c}{ Male } \\
& Low & High & Low & High \\
\hline Trustworthiness & 8 & 7 & $6^{\dagger}$ & 9 \\
Dominance & 9 & 9 & 7 & 10 \\
Both & 6 & 3 & $1^{*}$ & 6 \\
\hline
\end{tabular}

Note. All $p$-values are equal to or less than .001 , except $\dagger=.004, *=.077$.

This further exemplifies the issue with the method of extremes when used in a multivariate setting. Throwing away a large part of the data to create the composites is not ideal, but given correlated traits, the actual size of the candidate pool for any composite is likely to be far smaller than the rule of thumb. In practice, this means transforms are based on the appearance of a very small sample of individuals, with some appearances making into several composites. While this problem is not as pronounced across all composites - i.e., relatively few faces appear in all high or low composites (though still significantly), there is nonetheless significant overlap 
between at least two traits. The face regression approach stands to address these issues by using all of the available data, and leveraging the properties of regression by holding variance in appearance constant for variables not of interest.

\section{Face Regression on a multivariate dataset}

Here, the algorithm is used to study the relationships between perceived trustworthiness, dominance, and attractiveness, key facets of social perception (Sutherland et al., 2013). As before, each aspect of facial appearance - shape coordinates and pixel values - are modelled individually using separate multiple regressions, using the average rating of trustworthiness, dominance, and attractiveness assigned to the faces as predictors. Rather than build two models for female and male faces, coding female faces with a one and males as negative one specified an additional predictor. While not examined here, this approach offers the utility to investigate traits without the effect of sexual dimorphism, for example. In addition, before modelling took place, each predictor was standardised through $z$-scoring. Given that each predictor then has a mean of zero, it offers a unique interpretation of the final model - it represents the average face, plus or minus the effects of particular traits, since outcome measures take on the mean value when predictors are centred on zero. More formally, the model for this particular dataset can be interpreted as:

$$
\begin{aligned}
\text { Predicted Face } & =\text { Average Face }+\beta_{\text {Attractiveness }} X_{1}+\beta_{\text {Trustworthiness }} X_{2}+\beta_{\text {Dominance }} X_{3} \\
& +\beta_{\text {Sex }} X_{4}
\end{aligned}
$$

First, the regression approach was used to recreate the composite faces. If composite faces capture variation in correlated traits other than the dimension of interest, then the model should be able to recreate the composite faces exactly, as it did in Study One, by using the scores of all three traits as predictors The high and low composite faces were generated for each sex, for each trait, using the 15 high and low scorers. At the same, the mean $z$-score value for each trait that was entered into each 
composite was calculated and passed to the model. Subsamples of the model's predictions are shown in Figure 6 for Trustworthiness and Dominance. Again, the regression approach shows excellent performance in recreating the composite images. This indicates two things: First, the social traits of dominance and trustworthiness also vary in a linear fashion with appearance. Second, and most important from a theoretical perspective, is that composites definitely recruit additional covariation in traits. To further measure the difference in recreation, the root mean square error (RMSE) was computed for each of the high/low, female/male, and trait combinations, comparing the predicted face to the composite image. Error variability was very low across these comparisons, within approximately five pixel value units on average ( $M$ $=5.53, S D=0.33$ ). These results indicate that the method of extremes captures more variability than is of interest, and that facial transforms use more cues than the given dimension.
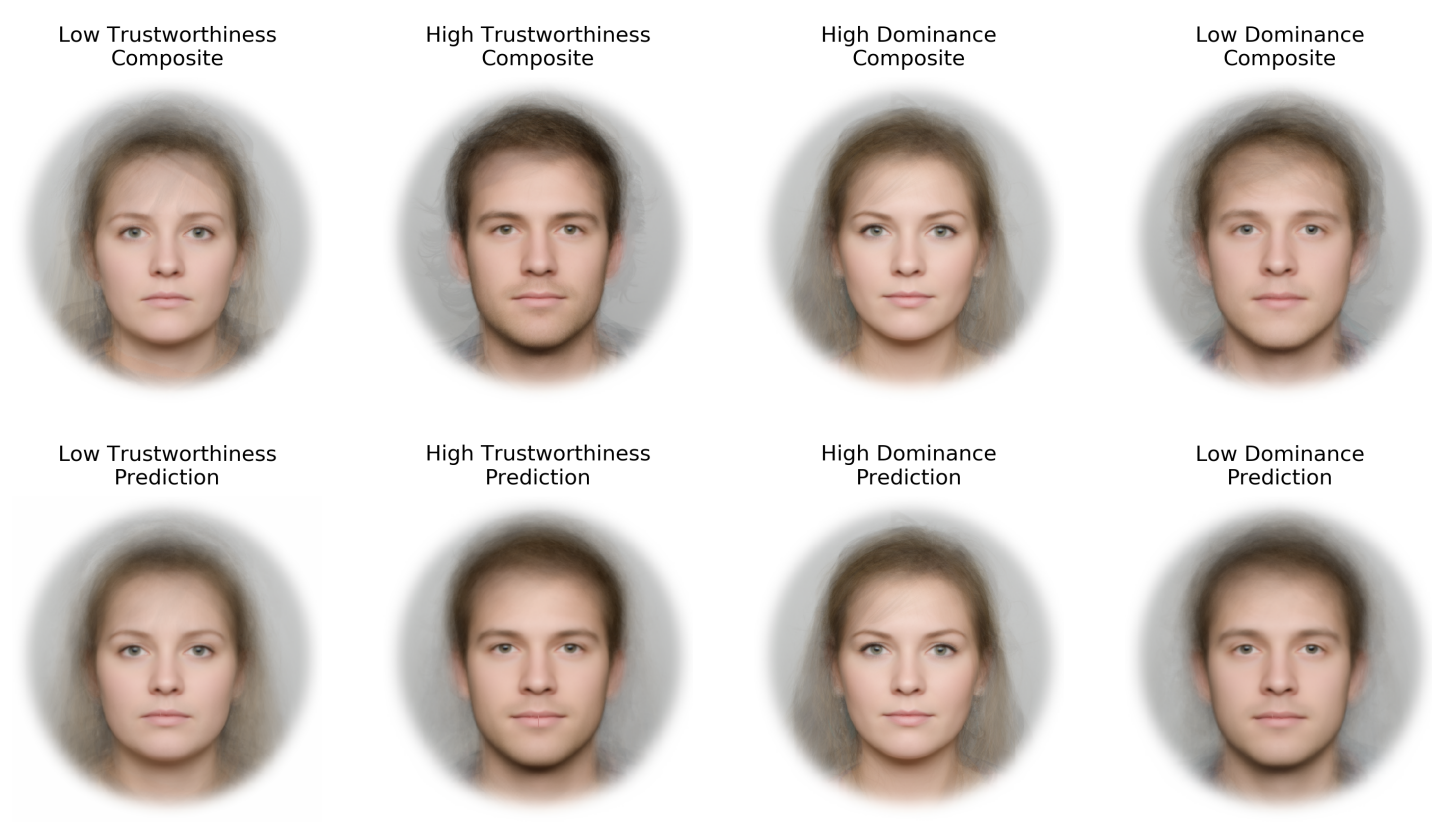
Figure 6. Examples of the model's ability to reconstruct an extreme composite image when the mean score of all measured traits that comprise the composite are passed to the model. The high degree of similarity is evidence that the method of extremes captures variance other than the trait of interest.

\section{Generating 'pure' appearances}

Multiple regression has the useful property that the regression weights of individual predictors are interpreted as the effect of one independent predictor on the outcome variable, holding all other predictors constant. This approach is mapped easily to the face regression approach, where the outcome is a facial appearance, and the influences of other traits are held constant. Here, the model is used to hold the influence of two of the three traits constant, while using the mean rating value of the high or low composite faces' as the weight to the predictor of interest. This process was carried out across all three traits, for both high and low levels, and for both sexes. The results are shown in Figure 7, comparing the different appearances of the composite faces (which captures variance across all traits) and the predicted face, which holds other traits constant. These are referred to as the 'pure' appearance of a trait. As predicted, the 'pure' faces have a different facial appearance to the composite faces, confirming the composites 'bake in' facial morphology uniquely associated with the other cues. 
Comparison of Attractiveness visualisations
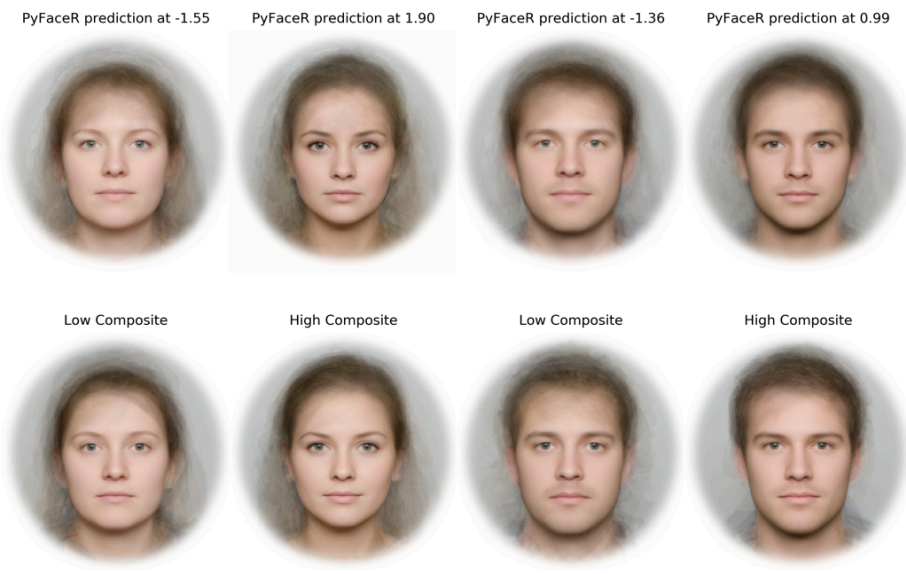

Comparison of Trustworthiness visualisations
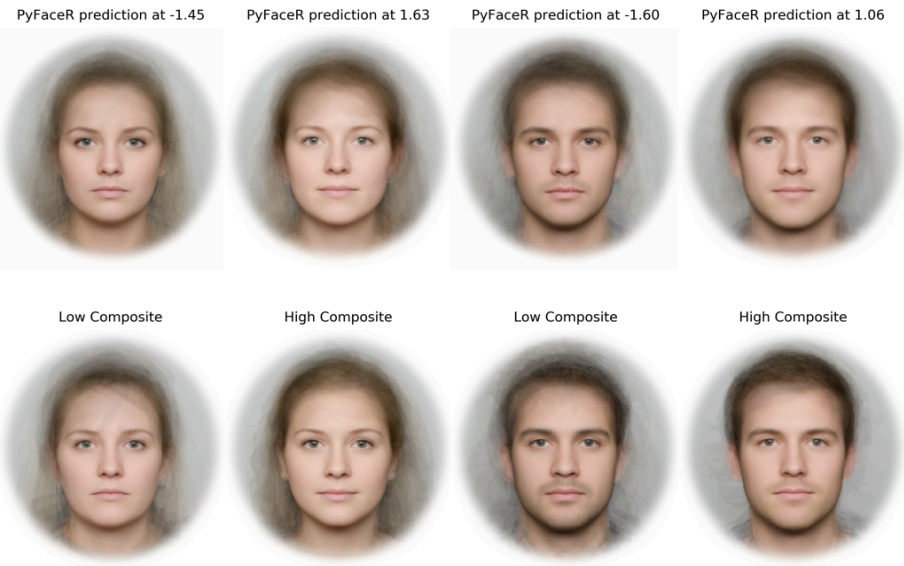

Comparison of Dominance visualisations
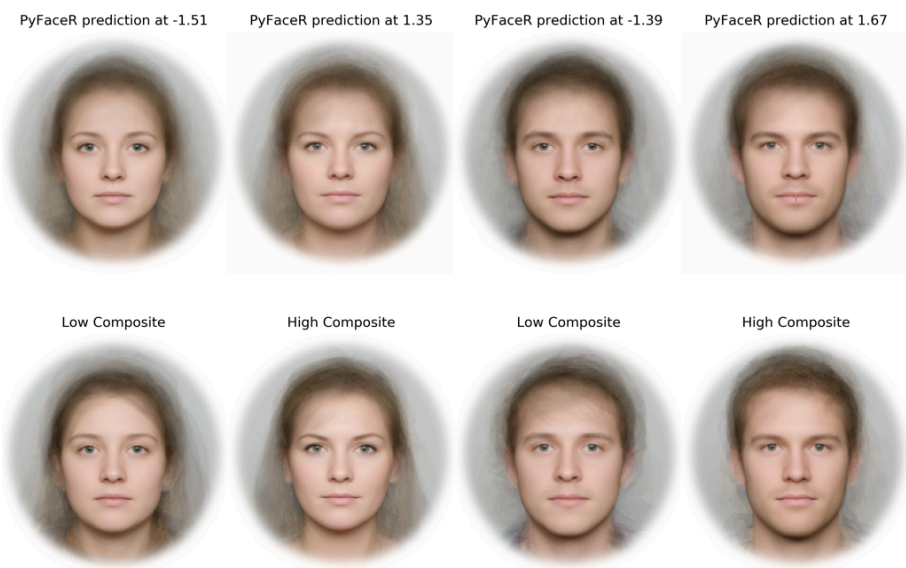

Figure 7. Comparison of facial appearances generated through the method of extremes, with the face regression approach holding constant two of the traits. The differences in appearance represent the 'pure' appearance of a particular trait, generated using the full dataset.

\section{Initial perceptual validation}


Given that the facial appearance of pure and composite faces is sufficiently different, a simple experiment was carried out to test observer responses to the images. Participants viewed pairs of pure and composite images, matched for sex and the high versus low level of the trait. Trials were blocked by trait, with each block containing only the comparisons for that trait. Participants were asked to select the face that appeared more attractive, trustworthy or dominant using a two alternative forced choice design, for a total of 12 trials. Participants viewed faces containing solely the information related to the trait of interest, comparing it to faces that contained the same information alongside correlated information - the composites.

A straightforward hypothesis is that if traits in social perception are correlated, then observers will select the composite face more often than the pure. That is, the extra information in the composites faces may be recruited to make a judgment of a singular trait, despite the unique appearance of that trait being present.

Participants. One hundred and ten participants ( 72 female, one other, age $M=$ $28.31, S D=11.46)$ were recruited for this study using Prolific.ac and sharing via social media sites. Participants followed a link to the study, implemented using the Gorilla (gorilla.sc) platform. Prolific participants were awarded $£ 0.25$ for their participation. Two participants were removed from the analysis for not completing all 12 trials.

Design. As discussed above, participants completed a 12 trial two alternative forced choice task between pure and composite faces, across three factors - trait level (high or low), sex (female or male) and the three traits (attractiveness, trustworthiness, dominance). Responses were coded as one for selecting a pure face, and zero for a composite face. The left to right ordering of the stimuli was counterbalanced between 
participants. Trials were blocked by trait, with each block appearing in a random order, and with trial order randomised within each block.

Results. To check for any differential responses between participants recruited from different sources, raw response data was submitted to an initial mixed model ANOVA, with three repeated measures factors (Sex, Level, and Trait) and one between measures factor (Participant Source: Prolific or Social Media). There were no main effects or interactions between Source and other variables, all $F \mathrm{~s}<2.07, p \mathrm{~s}>$ 0.12. As such, responses were collapsed across Source and reanalysed. This three factor repeated measures ANOVA revealed a three way interaction between Sex, Level, and Trait, $F(2,214)=12.85, p<.001, \eta^{2}=.02$. This interaction is shown in Figure 8 , and led to each trait being examined separately.
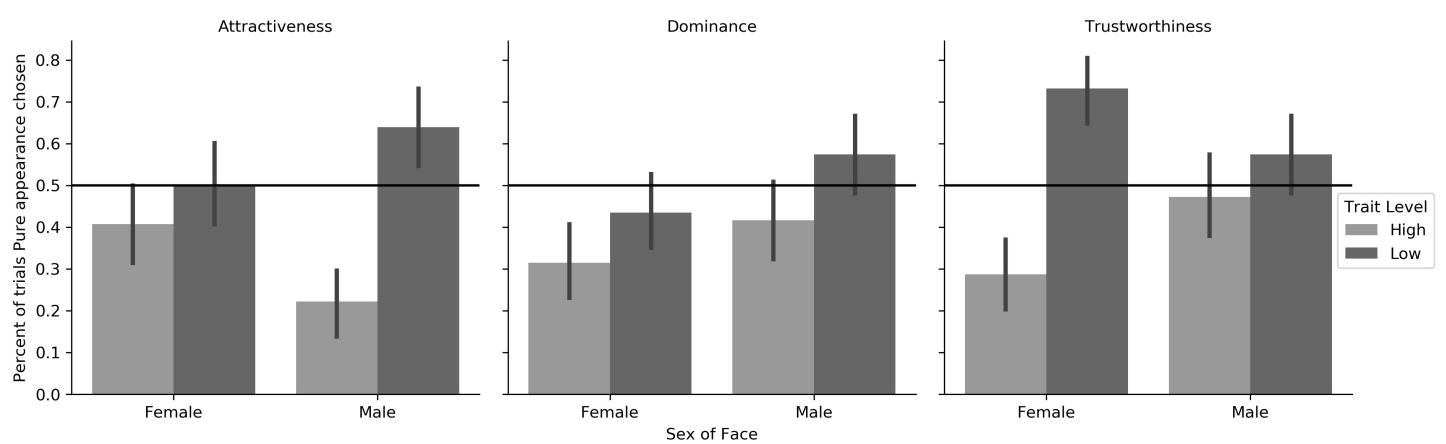

Figure 8. Means for each combination of Sex, Level, and Trait. Higher scores indicate a greater proportion of trials where the pure appearance was selected, as compared to the composite appearance. Error bars represent $95 \%$ confidence intervals. Solid black line represents chance $(0.50)$.

Attractiveness. A Sex by Level ANOVA for this trait revealed a significant interaction, $F(1,107)=11.94, p<.001, \eta^{2}=.03$. Observers showed no significant difference in selecting the pure appearance at between high $(M=.41)$ or low levels $(M=.50)$ of attractiveness for female faces, $t(107)=-1.37, p=.175, d=0.13$. For male faces, observers selected the pure appearance significantly more often for low attractiveness $(M=.64)$ as compared to high $(M=.22), t(107)=-6.59, p<.001, d=$ .64. While this provides evidence of perceptual differences between trait levels within 
the sex of faces, it is also worth comparing the absolute preference for the pure appearance over the composite for each combination of sex and level. To do this, each variable was compared to chance $(0.50)$ using a one sample $t$-test, revealing whether observers chose the pure or composite face as appearing more attractive. For high female attractiveness, there was a weak trend towards the composite face, $t(107)=$ $1.95, p=0.05, d=0.19$ but no difference for low female attractiveness, $t(107)=0.00$ $p=1, d=0.00$. For high male attractiveness, a significant bias towards the composite face was evident, $t(107)=-6.91, p<.001, d=0.67$, while for low male attractiveness, observers showed a significant bias towards the pure appearance, $t(107)=2.99, p=$ $.003, d=0.29$.

Dominance. A main effect of Sex (female $M=.38$, male $M=.49), F(1,107)=$ $6.21, p=.014, \eta^{2}=.02$, as well as Level (low $M=.50$, high $\left.M=.37\right), F(1,107)=$ $7.81, p=.006, \eta^{2}=.02$ emerged for dominance, indicating a stronger bias towards composite faces (i.e., lower scores) for female faces and more dominant appearances respectively. Comparing each variable to chance indicated a significant preference towards the composite image for high female dominance faces, $t(107)=-4.12, p<$ $.001, d=0.40$, but not for low female dominance, $t(107)=-1.35, p=.179, d=0.13$. A trend towards the pure appearance appeared for high male dominance, $t(107)=-$ $1.75, p=.083, d=0.17$, but not for low male dominance, $t(107)=1.55, p=.124, d=$ 0.15 .

Trustworthiness. A significant interaction between Sex and Level was present for the trait, $F(1,107)=13.52, p<.001, \eta^{2}=.03$. Observers selected the low female pure appearance $(M=.73)$ more often than the pure high appearance $(M=.29), t(107)$ $=-6.58, p<.001, d=0.64$, a pattern not present when comparing low male $(M=.57)$ and high male $(M=.47)$ trustworthiness, $t(107)=-1.55, p=.124, d=0.15$. 
Comparing each variable to chance revealed a significant bias towards the composite image for high female trustworthiness, $t(107)=-4.87, p<.001, d=0.47$, but a significant bias towards the pure appearance for low female trustworthiness, $t(107)=$ $5.40, p<.001, d=0.52$. No bias was present for high, $t(107)=-0.58, p=.566, d=$ 0.06 , or low male trustworthiness, $t(107)=1.55, p=.124, d=0.15$.

Discussion. The results suggest that observers respond differently to faces conveying the isolated, pure appearance of a trait when compared to a composite image that carries other correlated trait information. In some instances, no observer bias was evident between the composite and pure appearances (male trustworthiness and dominance, female attractiveness), indicating a relative focus on the appearance of the features related to the trait in question. However, in other conditions, significant differences emerged. For example, for male attractiveness, observers more often selected the composite face, indicating that the appearance of male attractiveness is linked to appearances other than the facial appearance solely linked to attractiveness. A similar pattern emerged for high female dominance and trustworthiness - observers chose the composite appearance over the pure, suggesting the extra trait information in the composites influences the perceptions of a trait, beyond the appearances solely related to the trait itself. Conversely, for low male attractiveness and low female trustworthiness, observers showed a bias to the pure appearance over the composite. As observers were asked to select the face they thought appeared higher on the trait, this suggests that the extra information present in the low composite faces in these conditions may be driving perceptions of the trait of interest. As an example, a male face low in attractiveness is seen as unattractive because of features that are not directly linked to attractiveness. 
These findings support the notions set out earlier, in that the method of extremes may capture more variation than is of interest. It also reveals that this variation may be responsible for driving social perceptions of traits more than the appearance directly associated with that trait. That is, faces carry extra information, unrelated to a particular trait, which may 'bias' a judgment of that trait. This has important implications for the approaches used in understanding social perception from faces, because this 'bias' information is inherently present in composite images and may be responsible for the responses made by observers. That is, the traits that captured using the method of extremes and subsequently presented or used to warp images may not be the trait of interest that observers are responding to.

\section{Second perceptual validation - examining 'bias' information}

The initial perceptual validation showed that in instances where a difference between the composite and pure faces are apparent, observers tend to perceive the composite face as appearing more like a given trait over a face that genuinely and solely conveys the appearance. That is, the extra, or bias information in the composite faces removed by the face regression approach may be carrying important social information beyond the actual appearance of the trait, which nonetheless contributes to the perception of that trait. Fortunately, the algorithm is able to recover this bias information just as easily. In the following study, this bias information is isolated using the face regression approach, and observer responses are collected when comparing this information to appearances containing theoretically no information an average face. This approach isolates the bias information, revealing what kind of traits an observer might perceive in it.

Participants. Ninety-two participants (53 female, age $M=30.20, S D=9.80$ ) were recruited for this study using Prolific.ac and sharing via social media sites. 
Participants followed a link the study, implemented using the Gorilla (gorilla.sc) platform. Prolific participants were awarded $£ 0.25$ for their participation.

Design. As before, participants completed a two alternative forced choice task, consisting of 12 trials, with one trial for each combination of Sex, Level, and Trait, with the same counterbalancing and randomisation procedures as before. To isolate the bias information for each trait, the 15 highest and lowest scoring faces from each sex and trait were taken, and the average score for all three traits was computed. Then, the mean value of the trait of interest was set to zero, but the mean values of the remaining traits were retained. For example, to isolate the bias information in a high trustworthiness composite, the mean score of trustworthiness, dominance, and attractiveness was computed. The value of trustworthiness was then set to zero, while retaining the scores for dominance and attractiveness. These were then passed to the model to predict the bias information appearance, essentially the reverse process to producing the pure appearance from the initial study. A conceptually simple way of understanding the approach is that the composite face is the sum of the pure appearance and the bias information. To generate the average faces, all traits were set to zero aside from the sex of the face to produce an average female or male appearance. Participants then selected between the average face and the sex and trait specific bias appearance on each trial as looking more like the trait. Responses were coded as one for a selection of the bias appearance, and zero for the average appearance.

Results. To check for any differential responses between participants recruited from different sources, raw response data was submitted to an initial mixed model ANOVA, with three repeated measures factors (Sex, Level, and Trait) and one between measures factor (Participant Source: Prolific or Social Media). There were 
no main effects or interactions between Source and other variables, all $F \mathrm{~s}<2.63, p \mathrm{~s}>$ 0.07. As such, responses were collapsed across Source and reanalysed. This three factor repeated measures ANOVA again revealed a three way interaction between Sex, Level, and Trait, $F(2,182)=11.34, p<.001, \eta^{2}=.02$. This interaction is shown in Figure 9. Each trait was then examined separately.

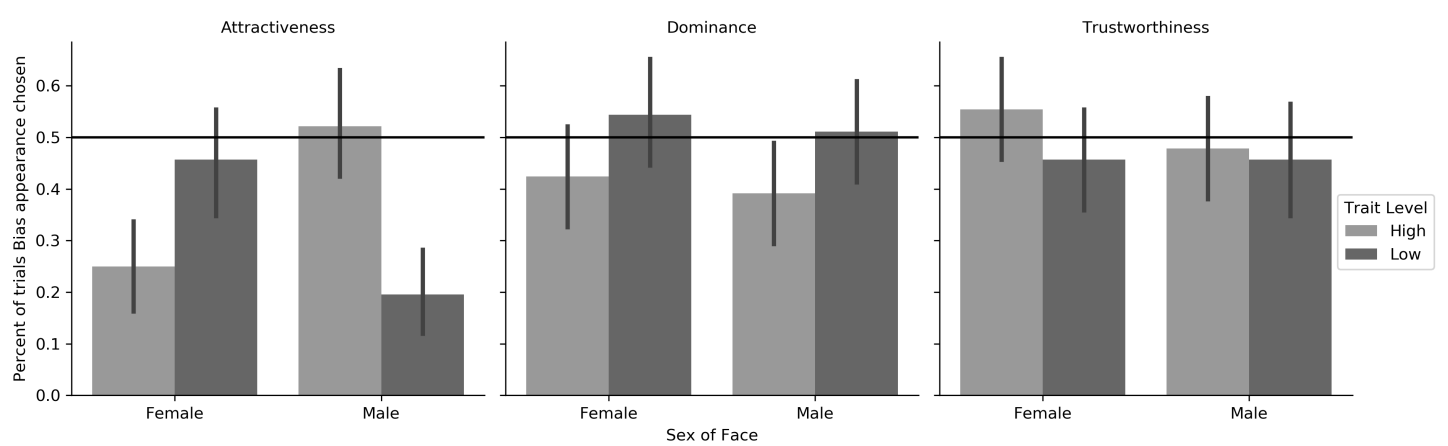

Figure 9. Means for each combination of Sex, Level, and Trait. Higher scores indicate a greater proportion of trials where the bias appearance was selected, as compared to the sex-matched average face. Error bars represent 95\% confidence intervals. Solid black line represents chance $(0.50)$.

Attractiveness. A Sex by Level ANOVA for this trait revealed a significant interaction, $F(1,91)=27.33, p<.001, \eta^{2}=.08$. Observers selected the average face as more attractive more frequently than the bias appearance for high female attractiveness $(M=.25)$ as compared to low $(M=.46), t(91)=-2.88, p=.005, d=$ 0.30. In contrast, observers selected the average face as more attractive with greater frequency for low male attractiveness $(M=.20)$ as compared to high $(M=.52), t(91)$ $=4.83, p<.001, d=0.51$. Each variable was also compared to chance $(0.50)$ using a one sample $t$-test, revealing whether observers chose the bias or average face as appearing more attractive. For high female attractiveness, observers chose the average face more often, $t(91)=-5.51, p<0.001, d=0.58$, but no preference was evident for low female attractiveness, $t(91)=-0.83, p=.407, d=0.09$. For high male attractiveness, no preference was evident, $t(91)=0.42, p=.679, d=0.04$, while for 
low male attractiveness, observers showed a significant bias towards the average male appearance, $t(91)=-7.32, p<.001, d=0.77$.

Dominance. The Sex by Level ANOVA revealed no significant main effects or an interaction, all $F \mathrm{~s}<3.35, p \mathrm{~s}>.070$. Comparing each variable for dominance to chance revealed no preference for the bias or average appearance for female high, $t(91)=-1.47, p=.145, d=0.15$, or low dominance, $t(91)=0.83, p=.407, d=0.09$. A small observer preference for the average face was evident for high male dominance, $t(91)=-2.12, p=.036, d=0.22$, but not for low, $t(91)=0.20, p=.836, d=0.02$.

Trustworthiness. The Sex by Level ANOVA revealed no significant main effects or interactions, all $F_{\mathrm{s}}<1.07, p \mathrm{~s}>.303$. Comparing each variable to chance also revealed no preferences between the bias and average appearance across all combinations of Sex and Level: high female $t(91)=1.04, p=.300, d=0.11$, low female $t(91)=-0.83, p=.407, d=0.09$, high male $t(91)=-0.42, p=.679, d=0.04$, low male $t(91)=-0.83, p=.407, d=0.09$.

Discussion. The results here indicate that, for attractiveness in particular, information in faces that is correlated with attractiveness can drive perceptions of that trait. For high female and low male attractiveness, observers selected the average face (i.e., no information regarding attractiveness) over the bias information, suggesting that the bias information is considered less attractive than an average face. That is, information in attractive female faces not directly linked to attractiveness may be misleading, possibly reducing the attractiveness of the face. Similarly, for low attractive male faces, the bias information was less attractive than the average face low attractive male faces may be made unattractive by this biased information. This particular finding is supported by those of the initial validation study, where observers preferred the pure appearance over the composite, suggesting it is the correlated, bias 
information that reduces attractiveness, rather than traits associated with attractiveness itself.

For other traits, there was no indications that bias information was preferred or not, when compared to the average face. This is in contrast to the findings of the initial validation experiment, where bias information may have been driving the perceptions. This opens up an intriguing possibility; at least for the case of attractiveness, trustworthiness, and dominance - that faces may be greater than the sum of their parts. That is, the pure appearance and the bias appearance may not be simply additive, forming the composite face, but could instead interact. That is, while a comparison between a pure and composite appearance may show a preference for the composite face (i.e., bias over pure appearance), the bias appearance alone is not sufficient to cause a perception of a trait, but may be necessary. Additionally, there may be other variables affecting perceptions that are not modelled here (e.g., intelligence) that could be further contributing to these perceptions.

The findings from both validation studies suggest that perceptions of social traits, typically captured in composites created from small samples of faces, contain more information than the traits they set out to capture. Observers may be reacting to this other information in these faces, whether they are presented as stimuli, or used to linearly transform other faces. While the traits examined here are central to social perception (Sutherland et al., 2013), all traits showed varying patterns with regards to observer responses to composite, pure, and bias information. This may indicate that social perceptions drawn from faces may have unique patterns of inference, drawn from combinations of appearances that are both directly linked to the trait as well as those that are not.

\section{General Discussion}


Advances in the field of face perception are linked to innovations in computational approaches that allow for a finer grained understanding of the nature of social judgments (Oosterhof \& Todorov, 2008; O’Toole et al., 1999; Tiddeman et al., 2001). The initial application of face regression here has demonstrated its utility in furthering the understanding of how social perceptions are drawn from faces through the application of linear models to high dimensional face data.

The algorithm was developed to address three criticisms of the general methodology used in face perception. The first was that typical approaches that rely on averaging of small subsets of extreme scorers as stimuli, or to produce stimuli, are characterised as 'data-driven' (Sutherland et al., 2017). The argument here is that this approach is not data driven, but is instead highly wasteful: inferences are made on small subsets of faces that are likely to overlap in the case of multiple trait studies. The face regression algorithm successfully overcomes this limitation by using all available data to create arrays of regression weights that model each dimension of available face data. Second, when creating composites using the method of extremes, an assumption is that the faces represent the appearance of a trait of interest, and that if any other traits are present, they are inconsequential. This assumption is likely flawed, given that the nature of perception is that almost all judgments are correlated to some degree (Brunswik, 1956; Todorov et al., 2008), which is supported by studies using morphometric approaches (Linke et al., 2016). The face regression algorithm is capable of using all available data to isolate the features associated with high or low levels of a trait (i.e. the pure appearance), whether they are shape or texture, by using multiple regression to hold constant appearances other than the one of interest. Indeed, the demonstration here revealed that, in some instances, these additional features are capable of biasing perceptions of the trait of interest, which has many 
theoretical implications. Third, the majority of current literature holds the assumption that perception of social traits covary linearly with facial appearance, based mostly on transforms developed from composites of small numbers of extreme scorers (Sutherland et al., 2015) or from complex analyses (Vernon et al., 2014). The face regression algorithm confirms that, at least for the traits examined here, that appearance varies linearly with perception - linear models are capable of recreating facial appearances that match the composites, illustrating they are 'snapshots' of appearances along a scale.

\section{Limitations}

The face regression approach allows for a more complete understanding of how facial appearance varies with social traits. Given it utilises linear models, the algorithm is limited in its effectiveness by two points. The first is that it will produce the best results when the relationship is linear. The extent to which these relationships are in fact linear is mostly unknown, but proxy measures applied in the literature indicate the algorithm can be applied to many existing relationships to study them in more detail, such as personality (Sutherland et al., 2015), health (Jones et al., 2016), and trustworthiness (Todorov et al., 2008). Second, the faces that can be predicted by the algorithm will be constrained to the limits of the data, given extrapolation is not a strength of linear regression (Snee, 1977). Predicting faces outside the range of the data is therefore unlikely to yield useful results. A similar outcome is clear with caricaturing, using image warping - eventually the results prove unusable (Sutherland et al., 2017). The face regression approach is explicit about when this will occur, which is around the limits of the observed data.

\section{Applications}


The face regression approach has already shown that composites of three traits fundamental to social perception contain additional information that may sway the judgments of observers. In addition, this 'bias information' alone is not always sufficient to alter observer preferences, indicating a kind of interaction with the actual appearances that influence trait judgments. A promising avenue of research is therefore applying the face regression approach to systematically vary the content present in faces and examining observer responses. Though not attempted here, it is entirely possible to remove one trait at a time and examine its impact - are high attractiveness faces still attractive if their trustworthiness is removed, for example? An additional possibility is to use the approach to produce adversarial appearances to test the relative values of cues to observers, something that is difficult to achieve with the method of extremes, given correlated traits. For example, how attractive might observers find a face that is high on attractiveness, but low on trustworthiness? Are there characteristics of observers that may make them overlook an undesirable trait for a positive one? This kind of approach is difficult to achieve in practice with the method of extremes without conflating image-warping steps, but is easily done here by passing positive and negative values to modelled attractiveness and trustworthiness weights. Another useful strategy would be to isolate sexual dimorphism. For example, dominant faces tend to be more masculine in appearance (Oosterhof \& Todorov, 2008; Quist, Watkins, Smith, DeBruine, \& Jones, 2011). To what extent is dominance associated with mere sexual dimorphism? The face regression approach can hold constant variation associated with sex, leaving just appearances (if any) associated with dominance that observers may rely on.

The approach could also benefit researchers investigating the relationships between appearance and actual, rather than perceived, traits. For example, facial 
composites of individuals high and low in actual Big 5 factors have been used to assess observer ability to accurately detect traits in faces (Jones et al., 2012; Kramer \& Ward, 2010; Little \& Perrett, 2007; Penton-Voak et al., 2006). However, individuals with extreme personality scores who are entered into these composites will also vary by default on other personality factors. The algorithm is capable of isolating a single trait for analysis, with a view to examining how other traits might influence a judgment. Extraverts may be judged as extraverted only because they are both extraverted and emotional stable, for example.

Another useful application could be in the realms of individual differences in perceptions. Research has highlighted that individuals with varying traits prefer different kinds of faces (Brown \& Sacco, 2016, 2017; Jones et al., 2007; Smith, Jones, \& DeBruine, 2010; Welling, Conway, Debruine, \& Jones, 2007; Welling, DeBruine, Little, \& Jones, 2009). To date, these approaches have mostly used image warping to produce pairs of images, typically the same identity, warped between composites (e.g. sexual dimorphism, actual personality), or just the composites themselves, and related the participants' choices to an individual difference measure. The face regression approach could offer a far more nuanced approach, similar to that of reverse correlation (Dotsch \& Todorov, 2012) to visualise individual differences in perception. Consider a set of faces rated by a variety of observers on a single trait. Each observer's ratings could be used as predictors in the algorithm, effectively visualising the face high or low on the trait, borne from the systematic ratings given by the observer. These faces could then be presented back to the observer for validation in a task. This method is easily extended to the case of groups - ratings of a set of faces could be averaged across raters with a given characteristic (e.g., extraverts) and the resulting faces compared to another group. If individual 
differences in face perception exist, this methodology would readily reveal them without the need for top down manipulations; for example, if more attractive women prefer more masculine faces (Vukovic et al., 2008), then the predicted face of attractive female raters should appear more masculine than that of less attractive raters. This approach also has advantages over reverse correlation: it uses real stimuli rather than noisy images, and, given enough variability in the initial sample of faces, may capture perceptions more accurately than the single stimuli that are used with reverse correlation (Jackson, Hester, \& Gray, 2018).

Finally, given the simple implementation of the algorithm (see supplementary materials), the predicted faces can be easily integrated with current face software packages like JPsychomorph (Tiddeman et al., 2001), allowing for warping, transforms, or averaging, measuring, or decomposition.

\section{Conclusion}

The face regression approach stands to bring a deeper understanding to how facial appearance is linked to the social world. Researchers have called for more refined measures that can help understand the complexities of 'psychomorphospace'

- the relationships between biology, facial appearance, and psychology (Schaefer, Mitteroecker, Fink, \& Bookstein, 2009). While many techniques have been used to study these relationships, each has had a downfall in that they focus on one aspect of appearance exclusively, uses data inefficiently, or, commonly, are technically inaccessible to the majority of researchers. Inspired by the history of techniques, here a new approach to understanding social perception from faces has been introduced, and benchmarked against well-established findings in the literature. The utility of face regression has been highlighted as a way to both visualise and analyse the relationships between facial appearance and social judgments, as well as 
demonstrating its ability to isolate variation to allow for more precision and control in understanding these relationships. Beyond these advantages, it has been implemented with a strong focus on interpretability and accessibility to researchers, who can apply it to great effect in the study of facial appearance and social perception. 


\section{References}

Adolphs, R., Nummenmaa, L., Todorov, A., \& Haxby, J. V. (2016). Data-driven approaches in the investigation of social perception. Philosophical Transactions of the Royal Society B: Biological Sciences, 371(1693). https://doi.org/10.1098/rstb.2015.0367

Balas, B., \& Pacella, J. (2017). Trustworthiness perception is disrupted in artificial faces. Computers in Human Behavior, 77, 240-248.

https://doi.org/10.1016/j.chb.2017.08.045

Bird, B. M., Geniole, S. N., Little, A. C., Moreau, B. J. P., Ortiz, T. L., Goldfarb, B., ... Carré, J. M. (2017). Does exogenous testosterone modulate men's ratings of facial dominance or trustworthiness? Adaptive Human Behavior and Physiology, 3(4), 365-385. https://doi.org/10.1007/s40750-017-0079-7

Boothroyd, L. G., Jones, B. C., Burt, D. M., DeBruine, L. M., \& Perrett, D. I. (2008). Facial correlates of sociosexuality. Evolution and Human Behavior, 29(3), 211-218. https://doi.org/10.1016/j.evolhumbehav.2007.12.009

Boothroyd, L. G., Jones, B. C., Burt, D. M., \& Perrett, D. I. (2007). Partner characteristics associated with masculinity, health and maturity in male faces. Personality and Individual Differences, 43(5), 1161-1173. https://doi.org/10.1016/j.paid.2007.03.008

Brown, M., \& Sacco, D. F. (2016). Avoiding extraverts: Pathogen concern downregulates preferences for extraverted faces. Evolutionary Psychological Science, 2(4), 278-286. https://doi.org/10.1007/s40806-016-0064-6

Brown, M., \& Sacco, D. F. (2017). Unrestricted sociosexuality predicts preferences for extraverted male faces. Personality and Individual Differences, 108, 123127. https://doi.org/10.1016/j.paid.2016.12.023 
Brunswik, E. (1956). Perception and the representative design of psychological experiments (2nd ed.). Berkeley: University of California Press.

Burt, D. M., \& Perrett, D. I. (1995). Perception of age in adult Caucasian male faces: computer graphic manipulation of shape and colour information. Proceedings of the Royal Society of London. Series B: Biological Sciences, 259(1355), 137-143. https://doi.org/10.1098/rspb.1995.0021

Butler, E. E., Saville, C. W. N., Ward, R., \& Ramsey, R. (2017). Physical attraction to reliable, low variability nervous systems: Reaction time variability predicts attractiveness. Cognition, 158, 81-89. https://doi.org/10.1016/j.cognition.2016.10.012

Chelnokova, O., Laeng, B., Eikemo, M., Riegels, J., Løseth, G., Maurud, H., ... Leknes, S. (2014). Rewards of beauty: The opioid system mediates social motivation in humans. Molecular Psychiatry, 19(7), 746-747. https://doi.org/10.1038/mp.2014.1

Davies-Thompson, J., Fletcher, K., Hills, C., Pancaroglu, R., Corrow, S. L., \& Barton, J. J. S. (2017). Perceptual learning of faces: A rehabilitative study of acquired prosopagnosia. Journal of Cognitive Neuroscience, 29(3), 573-591. https://doi.org/10.1162/jocn_a_01063

DeBruine, L. M., \& Jones, B. C. (2017). Face Research Lab London Set. figshare. Retrieved from https://doi.org/10.6084/m9.figshare.5047666.v3

DeBruine, L. M., Jones, B. C., Unger, L., Little, A. C., \& Feinberg, D. R. (2007). Dissociating averageness and attractiveness: attractive faces are not always average. Journal of Experimental Psychology. Human Perception and Performance, 33(6), 1420-1430. https://doi.org/10.1037/0096-1523.33.6.1420 
Deffenbacher, K. A., Vetter, T., Johanson, J., \& O’Toole, A. J. (1998). Facial Aging, Attractiveness, and Distinctiveness. Perception, 27(10), 1233-1243. https://doi.org/10.1068/p271233

Dotsch, R., \& Todorov, A. (2012). Reverse correlating social face perception. Social Psychological and Personality Science, 3(5), 562-571. https://doi.org/10.1177/1948550611430272

Farkas, L. G. (1987). Age and sex related changes in facial proportions. In Anthropometric facial proportions in medicine. Springfield IL: Charles C Thomas.

Fruhen, L. S., Watkins, C. D., \& Jones, B. C. (2015). Perceptions of facial dominance, trustworthiness and attractiveness predict managerial pay awards in experimental tasks. The Leadership Quarterly, 26(6), 1005-1016. https://doi.org/10.1016/j.leaqua.2015.07.001

George, P. A., \& Hole, G. J. (1995). Factors influencing the accuracy of age estimates of unfamiliar faces. Perception, 24(9), 1059-1073. https://doi.org/10.1068/p241059

Glassenberg, A. N., Feinberg, D. R., Jones, B. C., Little, A. C., \& DeBruine, L. M. (2010). Sex-dimorphic face shape preference in heterosexual and homosexual men and women. Archives of Sexual Behavior, 39(6), 1289-1296. https://doi.org/10.1007/s10508-009-9559-6

Henderson, A. J., Holzleitner, I. J., Talamas, S. N., \& Perrett, D. I. (2016). Perception of health from facial cues. Philosophical Transactions of the Royal Society B: Biological Sciences, 371(1693), 20150380. https://doi.org/10.1098/rstb.2015.0380 
Holtzman, N. S. (2011). Facing a psychopath: Detecting the dark triad from emotionally-neutral faces, using prototypes from the personality Faceaurus. Journal of Research in Personality, 45(6), 648-654. https://doi.org/10.1016/j.jrp.2011.09.002

Holzleitner, I. J., Hunter, D. W., Tiddeman, B. P., Seck, A., Re, D. E., \& Perrett, D. I. (2014). Men's Facial Masculinity: When (Body) Size Matters. Perception, 43(11), 1191-1202. https://doi.org/10.1068/p7673

Holzleitner, I. J., \& Perrett, D. I. (2016). Perception of strength from 3D faces is linked to facial cues of physique. Evolution and Human Behavior, 37(3), 217229. https://doi.org/10.1016/j.evolhumbehav.2015.11.004

Jackson, J. C., Hester, N., \& Gray, K. (2018). The faces of God in America: Revealing religious diversity across people and politics. PLOS ONE, 13(6), e0198745. https://doi.org/10.1371/journal.pone.0198745

Jones, A. L. (2018). The influence of shape and colour cue classes on facial health perception. Evolution and Human Behavior, 39(1), 19-29. https://doi.org/10.1016/j.evolhumbehav.2017.09.005

Jones, A. L., Kramer, R. S. S., \& Ward, R. (2012). Signals of personality and health: the contributions of facial shape, skin texture, and viewing angle. Journal of Experimental Psychology. Human Perception and Performance, 38(6), 13531361. https://doi.org/10.1037/a0027078

Jones, A. L., Kramer, R. S. S., \& Ward, R. (2014). Miscalibrations in judgements of attractiveness with cosmetics. Quarterly Journal of Experimental Psychology, 67(10), 2060-2068. https://doi.org/10.1080/17470218.2014.908932

Jones, A. L., Porcheron, A., Sweda, J. R., Morizot, F., \& Russell, R. (2016). Coloration in different areas of facial skin is a cue to health: The role of cheek 
redness and periorbital luminance in health perception. Body Image, 17, 5766. https://doi.org/10.1016/j.bodyim.2016.02.001

Jones, B. C., DeBruine, L. M., Little, A. C., Conway, C. A., Welling, L. L. M., \& Smith, F. (2007). Sensation seeking and men's face preferences. Evolution and Human Behavior, 28(6), 439-446.

https://doi.org/10.1016/j.evolhumbehav.2007.07.006

Jones, B. C., Hahn, A. C., Fisher, C. I., Wang, H., Kandrik, M., Han, C., ...

DeBruine, L. M. (2018). No compelling evidence that preferences for facial masculinity track changes in women's hormonal status. Psychological Science, 0956797618760197. https://doi.org/10.1177/0956797618760197

King, D. E. (2009). Dlib-ml: A Machine Learning Toolkit. Journal of Machine Learning Research, 10, 1755-1758.

Kleisner, K., Chvátalová, V., \& Flegr, J. (2014). Perceived intelligence is associated with measured intelligence in men but not women. PLOS ONE, 9(3), e81237. https://doi.org/10.1371/journal.pone.0081237

Kramer, R. S. S., Jenkins, R., \& Burton, A. M. (2017). InterFace: A software package for face image warping, averaging, and principal components analysis. Behavior Research Methods, 49(6), 2002-2011. https://doi.org/10.3758/s13428-016-0837-7

Kramer, R. S. S., \& Ward, R. (2010). Internal facial features are signals of personality and health. The Quarterly Journal of Experimental Psychology, 63(11), 22732287. https://doi.org/10.1080/17470211003770912

Langlois, J. H., \& Roggman, L. A. (1990). Attractive faces are only average. Psychological Science, 1(2), 115-121. https://doi.org/10.1111/j.14679280.1990.tb00079.x 
Linke, L., Saribay, S. A., \& Kleisner, K. (2016). Perceived trustworthiness is associated with position in a corporate hierarchy. Personality and Individual Differences, 99, 22-27. https://doi.org/10.1016/j.paid.2016.04.076

Little, A. C., Jones, B. C., \& DeBruine, L. M. (2011). Facial attractiveness: evolutionary based research. Philosophical Transactions of the Royal Society B: Biological Sciences, 366(1571), 1638-1659. https://doi.org/10.1098/rstb.2010.0404

Little, Anthony C., \& Perrett, D. I. (2007). Using composite images to assess accuracy in personality attribution to faces. British Journal of Psychology, 98(1), 111-126.

Little, Anthony C., Roberts, S. C., Jones, B. C., \& DeBruine, L. M. (2012). The perception of attractiveness and trustworthiness in male faces affects hypothetical voting decisions differently in wartime and peacetime scenarios. The Quarterly Journal of Experimental Psychology, 65(10), 2018-2032. https://doi.org/10.1080/17470218.2012.677048

Mayer, C., Windhager, S., Schaefer, K., \& Mitteroecker, P. (2017). BMI and WHR are reflected in female facial shape and texture: A geometric morphometric image analysis. PLOS ONE, 12(1), e0169336. https://doi.org/10.1371/journal.pone.0169336

Michael Burt, D., Kentridge, R. W., Good, J. M. ., Perrett, D. I., Tiddeman, B. P., \& Boothroyd, L. G. (2007). Q-cgi: new techniques to assess variation in perception applied to facial attractiveness. Proceedings of the Royal Society B: Biological Sciences, 274(1627), 2779-2784. https://doi.org/10.1098/rspb.2007.1037 
Oldmeadow, J. A., Sutherland, C. A. M., \& Young, A. W. (2013). Facial Stereotype Visualization Through Image Averaging. Social Psychological and Personality Science, 4(5), 615-623. https://doi.org/10.1177/1948550612469820

Oosterhof, N. N., \& Todorov, A. (2008). The functional basis of face evaluation. Proceedings of the National Academy of Sciences, 105(32), 11087-11092.

O’Toole, A. J., Price, T., Vetter, T., Bartlett, J. C., \& Blanz, V. (1999). 3D shape and 2D surface textures of human faces: the role of "averages" in attractiveness and age. Image and Vision Computing, 18(1), 9-19. https://doi.org/10.1016/S0262-8856(99)00012-8

Penton-Voak, I. (2011). In retreat from nature? Successes and concerns in Darwinian approaches to facial attractiveness. Journal of Evolutionary Psychology, 9(2), 173-193. https://doi.org/10.1556/JEP.9.2011.2.5

Penton-Voak, I. S., Pound, N., Little, A. C., \& Perrett, D. I. (2006). Personality judgments from natural and composite facial images: More evidence for a "kernel of truth" in social perception. Social Cognition, 24(5), 607-640. https://doi.org/10.1521/soco.2006.24.5.607

Perrett, D. I., Lee, K. J., Penton-Voak, I., Rowland, D., Yoshikawa, S., Burt, D. M., ... Akamatsu, S. (1998). Effects of sexual dimorphism on facial attractiveness. Nature, 394(6696), 884-887. https://doi.org/10.1038/29772

Perrett, D. I., May, K. A., \& Yoshikawa, S. (1994). Facial shape and judgements of female attractiveness. Nature, 368(6468), 239-242. https://doi.org/10.1038/368239a0 
Quist, M. C., Watkins, C. D., Smith, F. G., DeBruine, L. M., \& Jones, B. C. (2011). Facial masculinity is a cue to women's dominance. Personality and Individual Differences, 50(7), 1089-1093. https://doi.org/10.1016/j.paid.2011.01.032

Re, D. E., DeBruine, L. M., Jones, B. C., \& Perrett, D. I. (2013). Facial cues to perceived height influence leadership choices in simulated war and peace contexts. Evolutionary Psychology, 11(1), 89-103.

Rhodes, G. (2006). The evolutionary psychology of facial beauty. Annual Review of Psychology, 57(1), 199-226. https://doi.org/10.1146/annurev.psych.57.102904.190208

Rohlf, F. J. (2015). The tps series of software. Hystrix, the Italian Journal of Mammalogy, 26(1), 9-12. https://doi.org/10.4404/hystrix-26.1-11264

Russell, R. (2009). A sex difference in facial contrast and its exaggeration by cosmetics. Perception, 38(8), 1211-1219. https://doi.org/10.1068/p6331

Sagonas, C., Antonakos, E., Tzimiropoulos, G., Zafeiriou, S., \& Pantic, M. (2016). 300 Faces In-The-Wild Challenge: Database and results. Image and Vision Computing, 47, 3-18. https://doi.org/10.1016/j.imavis.2016.01.002

Schaefer, K., Mitteroecker, P., Fink, B., \& Bookstein, F. L. (2009). Psychomorphospace-From biology to perception, and back: Towards an integrated quantification of facial form variation. Biological Theory, 4(1), 98106. https://doi.org/10.1162/biot.2009.4.1.98

Scott, N. J., Jones, A. L., Kramer, R. S. S., \& Ward, R. (2015). Facial dimorphism in Autistic Quotient scores. Clinical Psychological Science, 3(2), 230-241. https://doi.org/10.1177/2167702614534238 
Scott, N. J., Kramer, R. S. S., Jones, A. L., \& Ward, R. (2013). Facial cues to depressive symptoms and their associated personality attributions. Psychiatry Research, 208(1), 47-53. https://doi.org/10.1016/j.psychres.2013.02.027

Secord, P. (1958). Facial features and inference processes in interpersonal perception. In R. Taiguri \& L. Petrullo (Eds.), Person perception and interpersonal behavior (pp. 300-315). Stanford: Stanford University Press.

Sheehan, M. J., \& Nachman, M. W. (2014). Morphological and population genomic evidence that human faces have evolved to signal individual identity. Nature Communications, 5, 4800. https://doi.org/10.1038/ncomms5800

Smith, F. G., Jones, B. C., \& DeBruine, L. M. (2010). Individual differences in empathizing and systemizing predict variation in face preferences. Personality and Individual Differences, 49(6), 655-658.

https://doi.org/10.1016/j.paid.2010.05.023

Smith, F. G., Jones, B. C., DeBruine, L. M., \& Little, A. C. (2009). Interactions between masculinity-femininity and apparent health in face preferences. Behavioral Ecology, 20(2), 441-445. https://doi.org/10.1093/beheco/arn141

Snee, R. D. (1977). Validation of Regression Models: Methods and Examples. Technometrics, 19(4), 415-428. https://doi.org/10.1080/00401706.1977.10489581

Sofer, C., Dotsch, R., Wigboldus, D. H. J., \& Todorov, A. (2015). What is typical is good: the influence of face typicality on perceived trustworthiness. Psychological Science, 26(1), 39-47. https://doi.org/10.1177/0956797614554955

Sprengelmeyer, R., Young, A. W., Baldas, E.-M., Ratheiser, I., Sutherland, C. A. M., Müller, H.-P., ... Orth, M. (2016). The neuropsychology of first impressions: 
Evidence from Huntington's disease. Cortex, 85, 100-115. https://doi.org/10.1016/j.cortex.2016.10.006

Stephen, I. D., Coetzee, V., \& Perrett, D. I. (2011). Carotenoid and melanin pigment coloration affect perceived human health. Evolution and Human Behavior, 32(3), 216-227. https://doi.org/10.1016/j.evolhumbehav.2010.09.003

Sutherland, C. A. M., Oldmeadow, J. A., Santos, I. M., Towler, J., Michael Burt, D., \& Young, A. W. (2013). Social inferences from faces: Ambient images generate a three-dimensional model. Cognition, 127(1), 105-118. https://doi.org/10.1016/j.cognition.2012.12.001

Sutherland, C. A. M., Rhodes, G., \& Young, A. W. (2017). Facial image manipulation: A tool for investigating social perception. Social Psychological and Personality Science, 8(5), 538-551. https://doi.org/10.1177/1948550617697176

Sutherland, C. A. M., Rowley, L. E., Amoaku, U. T., Daguzan, E., Kidd-Rossiter, K. A., Maceviciute, U., \& Young, A. W. (2015). Personality judgments from everyday images of faces. Frontiers in Psychology, 6. https://doi.org/10.3389/fpsyg.2015.01616

Tiddeman, B., Burt, D. M., \& Perrett, D. (2001). Prototyping and transforming facial textures for perception research. IEEE Computer Graphics and Applications, 21, 42-50. https://doi.org/10.1109/38.946630

Todorov, A., Dotsch, R., Porter, J. M., Oosterhof, N. N., \& Falvello, V. B. (2013). Validation of data-driven computational models of social perception of faces. Emotion, 13(4), 724-738. https://doi.org/10.1037/a0032335 
Todorov, A., Said, C. P., Engell, A. D., \& Oosterhof, N. N. (2008). Understanding evaluation of faces on social dimensions. Trends in Cognitive Sciences, 12(12), 455-460. https://doi.org/10.1016/j.tics.2008.10.001

Vernon, R. J. W., Sutherland, C. A. M., Young, A. W., \& Hartley, T. (2014).

Modeling first impressions from highly variable facial images. Proceedings of the National Academy of Sciences, 111(32), E3353-E3361. https://doi.org/10.1073/pnas.1409860111

Vuilleumier, P., Armony, J. L., Driver, J., \& Dolan, R. J. (2003). Distinct spatial frequency sensitivities for processing faces and emotional expressions. Nature Neuroscience, 6(6), 624-631. https://doi.org/10.1038/nn1057

Vukovic, J., Feinberg, D. R., Jones, B. C., DeBruine, L. M., Welling, L. L. M., Little, A. C., \& Smith, F. G. (2008). Self-rated attractiveness predicts individual differences in women's preferences for masculine men's voices. Personality and Individual Differences, 45(6), 451-456. https://doi.org/10.1016/j.paid.2008.05.013

Welling, L. L. M., Conway, C. A., Debruine, L. M., \& Jones, B. C. (2007). Perceived vulnerability to disease is positively related to the strength of preferences for apparent health in faces. Journal of Evolutionary Psychology, 5(1), 131-139. https://doi.org/10.1556/JEP.2007.1012

Welling, Lisa L. M., DeBruine, L. M., Little, A. C., \& Jones, B. C. (2009).

Extraversion predicts individual differences in women's face preferences. Personality and Individual Differences, 47(8), 996-998. https://doi.org/10.1016/j.paid.2009.06.030

Windhager, S., Bookstein, F. L., Mueller, H., Zunner, E., Kirchengast, S., \& Schaefer, K. (2018). Calibrating facial morphs for use as stimuli in biological studies of 
social perception. Scientific Reports, 8(1), 6698.

https://doi.org/10.1038/s41598-018-24911-0

Wolffhechel, K., Hahn, A. C., Jarmer, H., Fisher, C. I., Jones, B. C., \& DeBruine, L. M. (2015). Testing the utility of a data-driven approach for assessing BMI from face images. PLOS ONE, 10(10), e0140347. https://doi.org/10.1371/journal.pone.0140347

Zebrowitz, L. A., \& Rhodes, G. (2004). Sensitivity to "bad genes" and the anomalous face overgeneralization effect: Cue validity, cue utilization, and accuracy in judging intelligence and health. Journal of Nonverbal Behavior, 28(3), 167185. https://doi.org/10.1023/B:JONB.0000039648.30935.1b 